\title{
1000 au exterior arcs connected to the protoplanetary disk around HL Tauri
}

\author{
Hsi-Wei Yen ${ }^{1}$, Shigehisa Takakuwa ${ }^{2,3}$, You-Hua $\mathrm{Chu}^{3}$, Naomi Hirano ${ }^{3}$, Paul T. P. Ho ${ }^{3,4}$, Kazuhiro D. Kanagawa ${ }^{5}$ \\ Chin-Fei Lee ${ }^{3}$, Hauyu Baobab Liu ${ }^{1}$, Sheng-Yuan $\mathrm{Liu}^{3}$, Tomoaki Matsumoto ${ }^{6}$, Satoki Matsushita ${ }^{3}$, Takayuki Muto ${ }^{7}$, \\ Kazuya Saigo ${ }^{8}$, Ya-Wen Tang ${ }^{3}$, Alfonso Trejo ${ }^{3}$, and Chun-Ju Wu ${ }^{9,3}$ \\ ${ }^{1}$ European Southern Observatory (ESO), Karl-Schwarzschild-Str. 2, 85748 Garching, Germany \\ e-mail: hyen@eso.org \\ 2 Department of Physics and Astronomy, Graduate School of Science and Engineering, Kagoshima University, 1-21-35 Korimoto, \\ Kagoshima, 890-0065 Kagoshima, Japan \\ 3 Academia Sinica Institute of Astronomy and Astrophysics, PO Box 23-141, 10617 Taipei, Taiwan \\ 4 East Asian Observatory, 660 N. A'ohoku Place, University Park, Hilo, HI 96720, USA \\ 5 Institute of Physics and CASA*, Faculty of Mathematics and Physics, University of Szczecin, Wielkopolska 15, 70-451 Szczecin, \\ Poland \\ ${ }^{6}$ Faculty of Sustainability Studies, Hosei University, Chiyoda-ku, 102-8160 Tokyo, Japan \\ 7 Division of Liberal Arts, Kogakuin University, 1-24-2 Nishi-Shinjuku, Shinjuku-ku, 163-8677 Tokyo, Japan \\ 8 ALMA Project Office, National Astronomical Observatory of Japan, Osawa 2-21-1, Mitaka, 181-8588 Tokyo, Japan \\ 9 Department of Physics, National Taiwan University, 10617 Taipei, Taiwan
}

Received 30 March 2017 / Accepted 4 August 2017

\begin{abstract}
Aims. The protoplanetary disk around HL Tau is the youngest candidate of planet formation known to date, and it is still embedded in a protostellar envelope with a size of thousands of au. In this work, we study the gas kinematics in the envelope and its possible influence on the embedded disk.

Methods. We present our new ALMA cycle 3 observational results of HL Tau in the ${ }^{13} \mathrm{CO}(2-1)$ and $\mathrm{C}^{18} \mathrm{O}(2-1)$ emission at resolutions of 0 '!8 (110 au), and we compare the observed velocity pattern with models of different types of gas motion.

Results. The ${ }^{13} \mathrm{CO}$ and $\mathrm{C}^{18} \mathrm{O}$ emission lines both show a central compact component with a size of $2^{\prime \prime}(280$ au) that traces the protoplanetary disk. The disk is clearly resolved and shows Keplerian motion, based on which, the protostellar mass of HL Tau is estimated to be $1.8 \pm 0.3 M_{\odot}$, assuming the inclination angle of the disk to be $47^{\circ}$ from the plane of the sky. The ${ }^{13} \mathrm{CO}$ emission shows two arc structures with sizes of 1000-2000 au and masses of $3 \times 10^{-3} M_{\odot}$ connected to the central disk. One is blueshifted and stretches from the northeast to the northwest, and the other is redshifted and stretches from the southwest to the southeast. We find that simple kinematical models of infalling and (counter-)rotating flattened envelopes cannot fully explain the observed velocity patterns in the arc structures. The gas kinematics of the arc structures can be better explained with three-dimensional infalling or outflowing motions. Nevertheless, the observed velocity in the northwestern part of the blueshifted arc structure is $\sim 60-70 \%$ higher than the expected free-fall velocity. We discuss two possible origins of the arc structures: (1) infalling flows externally compressed by an expanding shell driven by XZ Tau; and (2) outflowing gas clumps caused by gravitational instabilities in the protoplanetary disk around HL Tau.
\end{abstract}

Key words. protoplanetary disks - stars: formation - ISM: kinematics and dynamics

\section{Introduction}

Observations in (sub-)millimeter continuum at spatial resolutions of a few au and high-contrast imaging in the infrared have revealed rings and gaps with widths of a few au to tens of au in several protoplanetary disks (Akiyama et al. 2015; Momose et al. 2015; Rapson et al. 2015; Isella et al. 2016; Andrews et al. 2016; Ginski et al. 2016; Konishi et al. 2016; Perrot et al. 2016; van der Plas et al. 2017). The presence of these gaps suggests decreases in surface density or changes in dust properties at the location of these gaps (Takahashi \& Inutsuka 2014; Dipierro et al. 2015; Dong et al. 2015, 2016; Kanagawa et al. 2015, 2016; Tamayo et al. 2015; Zhang et al. 2015, 2016; Jin et al. 2016; Okuzumi et al. 2016; Dong \& Fung 2017). Hints of decreases in gas density coincident with the dust continuum gaps have also been observed in protoplanetary disks (Yen et al. 2016; Isella et al. 2016); this is suggestive of actual deficits in material at these gaps. Theoretical studies show that embedded gas giant planets can carve such gaps in protoplanetary disks (e.g., Dong et al. 2015, 2016; Kanagawa et al. 2015, 2016; Dong \& Fung 2017). Thus, these protoplanetary disks with rings and gaps are considered candidates of ongoing planet formation.

The protostar HL Tau $\left(\mathrm{J} 200004^{\mathrm{h}} 31^{\mathrm{m}} 38^{\mathrm{s}} .43+18^{\mathrm{d}} 13^{\mathrm{m}} 57^{\mathrm{s}} .2\right.$; ALMA Partnership et al. 2015) has a Class I-II spectral energy distribution (Men'shchikov et al. 1999) and is located in the Taurus star-forming region at a distance of $140 \mathrm{pc}$ (Kenyon et al. 1994; Loinard 2013). A series of rings and gaps were observed in its protoplanetary disk in the (sub-)millimeter continuum emission with the Atacama Large Millimeter/Submillimeter Array (ALMA), making HL Tau the youngest candidate of planet formation known to date (ALMA Partnership et al. 2015; Akiyama et al. 2016). Direct imaging in the $L^{\prime}$ band with the 
Large Binocular Telescope Interferometer did not find any point sources in the protoplanetary disk around HL Tau and placed an upper limit on the mass of possible planets of 10-15 $M_{\text {jup }}$ (Testi et al. 2015). The protoplanetary disk around HL Tau has an inclination angle of $47^{\circ}$ from the plane of the sky and a position angle (PA) of $138^{\circ}$ (ALMA Partnership et al. 2015), and it is embedded in an elongated molecular cloud with a length of $\sim 0.05$ pc (Welch et al. 2000). Observations in the ${ }^{13} \mathrm{CO}$ (10 ) emission at an angular resolution of $5^{\prime \prime}$ with the Nobeyama Millimeter Array show a flattened envelope with signs of infalling and rotational motions on a scale of 2000 au around HL Tau (Hayashi et al. 1993). Later observations in the ${ }^{13} \mathrm{CO}$ (1$0)$ emission with the Plateau de Bure interferometer (PdBI) and a combination of the BIMA and the NRAO $12 \mathrm{~m}$ telescope at an angular resolution of $3^{\prime \prime}-8^{\prime \prime}$ reveal complex velocity structures on a scale of hundreds to thousands of au (Cabrit et al. 1996; Welch et al. 2000). Thus, the gas motions in the envelope around HL Tau are still not clear. HL Tau is also associated with a bipolar molecular outflow (Monin et al. 1996; Lumbreras \& Zapata 2014; ALMA Partnership et al. 2015; Klaassen et al. 2016) and optical and infrared jets (Takami et al. 2007; Hayashi \& Pyo 2009; Anglada et al. 2007; Movsessian et al. 2012). The jets associated with HL Tau are moving at tangential velocities of 60$160 \mathrm{~km} \mathrm{~s}^{-1}$ with an inclination angle of $40^{\circ}-60^{\circ}$ and a PA of $35^{\circ}-$ $47^{\circ}$, measured from the proper motion of the jets (Anglada et al. 2007; Movsessian et al. 2012). The jet axis is perpendicular to the protoplanetary disk. Therefore, different from other candidates of planet formation, HL Tau is still surrounded by an protostellar envelope and is likely in the active mass accretion phase.

Dynamical infall from protostellar envelopes onto protoplanetary disks can produce accretion shocks in the disks (e.g., Yorke \& Bodenheimer 1999). This can change the chemical status of protoplanetary disks and possibly affect the subsequent chemical evolution of disks (e.g., Visser et al. 2009, 2011). Signs of accretion shocks have been observed in disks around Class 0 and I protostars, such as L1489 IRS (Yen et al. 2014), L1527 (Ohashi et al. 2014; Sakai et al. 2014a,b), and TMC-1A (Sakai et al. 2016). Changes in chemical compositions have also been observed in the transitional region between the infalling envelope and the central disk in L1527 (Sakai et al. 2014a,b) and IRAS 16293-2422 (Oya et al. 2016). In addition, as a protoplanetary disk continues to accrete mass from its surrounding envelope, it can become massive and gravitationally unstable (e.g., Machida et al. 2010; Vorobyov 2010). The gravitational instability can cause fragmentation and form gas clumps in disks, which can be the progenitors of planets (e.g., Vorobyov 2011; Zhu et al. 2012), and it can also trigger mass ejection from disks (e.g., Vorobyov 2016). These influences of protostellar envelopes on embedded protoplanetary disks are not well understood observationally.

These gaps and rings in the protoplanetary disk around HL Tau suggest that planet formation may occur when protoplanetary disks are still embedded in protostellar envelopes. To observationally investigate possible influences of protostellar envelopes on planet-forming disks, we have conducted ALMA observations in ${ }^{13} \mathrm{CO}(2-1 ; 220.398684 \mathrm{GHz})$ and $\mathrm{C}^{18} \mathrm{O}(2-1$; $219.560358 \mathrm{GHz})$ at an angular resolution of 0 '.8 (110 au) toward the candidate disk of planet formation around HL Tau. Our ALMA observations allow us to resolve the Keplerian rotation of the embedded protoplanetary disk and distinguish the gas motions in the surrounding envelope, and thus to study their relation. This paper is organized as follows: Sect. 2 describes the details of the observations. Section 3 presents the observational results in the ${ }^{13} \mathrm{CO}$ and $\mathrm{C}^{18} \mathrm{O}$ emission. Section 4 presents our analysis on the disk rotation traced by the ${ }^{13} \mathrm{CO}$ and $\mathrm{C}^{18} \mathrm{O}$ emission and on the kinematics of the surrounding structures connected to the disk observed in the ${ }^{13} \mathrm{CO}$ emission. Section 5 discusses the origins of the observed structures and kinematics in the ${ }^{13} \mathrm{CO}$ emission.

\section{Observations}

ALMA cycle- 3 observations toward HL Tau were conducted on 22 March, 2016 with 40 antennas. HL Tau was observed with a hexagonal seven-pointing mosaic with Nyquist sampling, and the total integration time on HL Tau is $30 \mathrm{~min}$. J0510+1800 with a flux of $2.38 \mathrm{Jy}$ at $225.7 \mathrm{GHz}$ was observed as bandpass and flux calibrator, and J0431+2037 with a flux of $65 \mathrm{mJy}$ at $225.6 \mathrm{GHz}$ as phase calibrator. The baseline length ranges from $14 \mathrm{~m}$ to $384 \mathrm{~m}$, so the maximum recoverable scale of our observations is $12^{\prime \prime}(1700 \mathrm{au})$. The typical absolute flux uncertainty of ALMA observations at $1 \mathrm{~mm}$ wavelength is $10 \%$. The spectral setup of our ALMA observations contained five spectral windows. Two spectral windows, each with a bandwidth of $2 \mathrm{GHz}$, were assigned to the $1.3 \mathrm{~mm}$ continuum. One spectral window with a bandwidth of $468.8 \mathrm{MHz}$ and a channel width of $122 \mathrm{kHz}$ was set to the ${ }^{13} \mathrm{CO}(2-1)$ line, one with a bandwidth of $234.4 \mathrm{MHz}$ and a channel width of $244 \mathrm{kHz}$ to the $\mathrm{C}^{18} \mathrm{O}(2-1)$ line, and one with a bandwidth of $234.4 \mathrm{MHz}$ and a channel width of $488 \mathrm{kHz}$ to the SO $\left(5_{6}-4_{5}\right)$ line. The raw visibility data were calibrated using the standard reduction script for the cycle-3 data, which uses tasks in the Common Astronomy Software Applications (CASA; McMullin et al. 2007) of version 4.6, and without self-calibration. We additionally flagged the data in the spectral window of the ${ }^{13} \mathrm{CO}$ line in field 4 at a time from 21:40:14 to 21:40:16 manually, because artificial stripes appear in images that are produced with uniform weighting. The image cubes of the ${ }^{13} \mathrm{CO}(2-1)$ and $\mathrm{C}^{18} \mathrm{O}(2-1)$ lines were generated with uniform weighting and cleaned with the CASA task "clean" at a velocity resolution of $0.34 \mathrm{~km} \mathrm{~s}^{-1}$. The angular resolution and the noise level are $0^{\prime \prime} .77 \times 00^{\prime \prime} .75$ and $7 \mathrm{mJy} \mathrm{Beam}^{-1}$ per channel in the ${ }^{13} \mathrm{CO}$ image, and 0 !.77 $\times 0$ 0'.74 and $6.7 \mathrm{mJy} \mathrm{Beam}^{-1}$ per channel in the $\mathrm{C}^{18} \mathrm{O}$ image.

\section{Results}

Figure 1 presents the total integrated-intensity (moment 0 ) maps overlaid on the intensity-weighted mean velocity (moment 1 ) maps of the ${ }^{13} \mathrm{CO}(2-1)$ and $\mathrm{C}^{18} \mathrm{O}(2-1)$ emission. The $\mathrm{C}^{18} \mathrm{O}$ emission primarily traces a compact component with an apparent size of $\sim 2^{\prime \prime}(280 \mathrm{au})$ around HL Tau, and it is oriented in the northwest-southeast direction with a PA of $\sim 125^{\circ}$. This orientation is consistent with that of the protoplanetary disk around HL Tau observed with ALMA at an angular resolution of $0^{\prime \prime}$.03 within $15^{\circ}$ (ALMA Partnership et al. 2015; Akiyama et al. 2015). The $\mathrm{C}^{18} \mathrm{O}$ central component exhibits a velocity gradient along its major axis, where the northwestern part is redshifted and the southeastern part is blueshifted. The direction of this velocity gradient is the same as the Keplerian rotation of the protoplanetary disk around HL Tau observed with ALMA in the $\mathrm{HCO}^{+}(1-0)$ and $\mathrm{CO}(1-0)$ emission at angular resolutions of $0^{\prime \prime} .2-0^{\prime \prime} .3$ (Pinte et al. 2016). The ${ }^{13} \mathrm{CO}$ emission also shows a similar central compact component, which is orientated and exhibits a velocity gradient in the northwest-southeast direction. In the ${ }^{13} \mathrm{CO}$ emission, there are additional extended structures with sizes of 1000-3000 au linking the central component. The extended structures stretch toward the northwest 

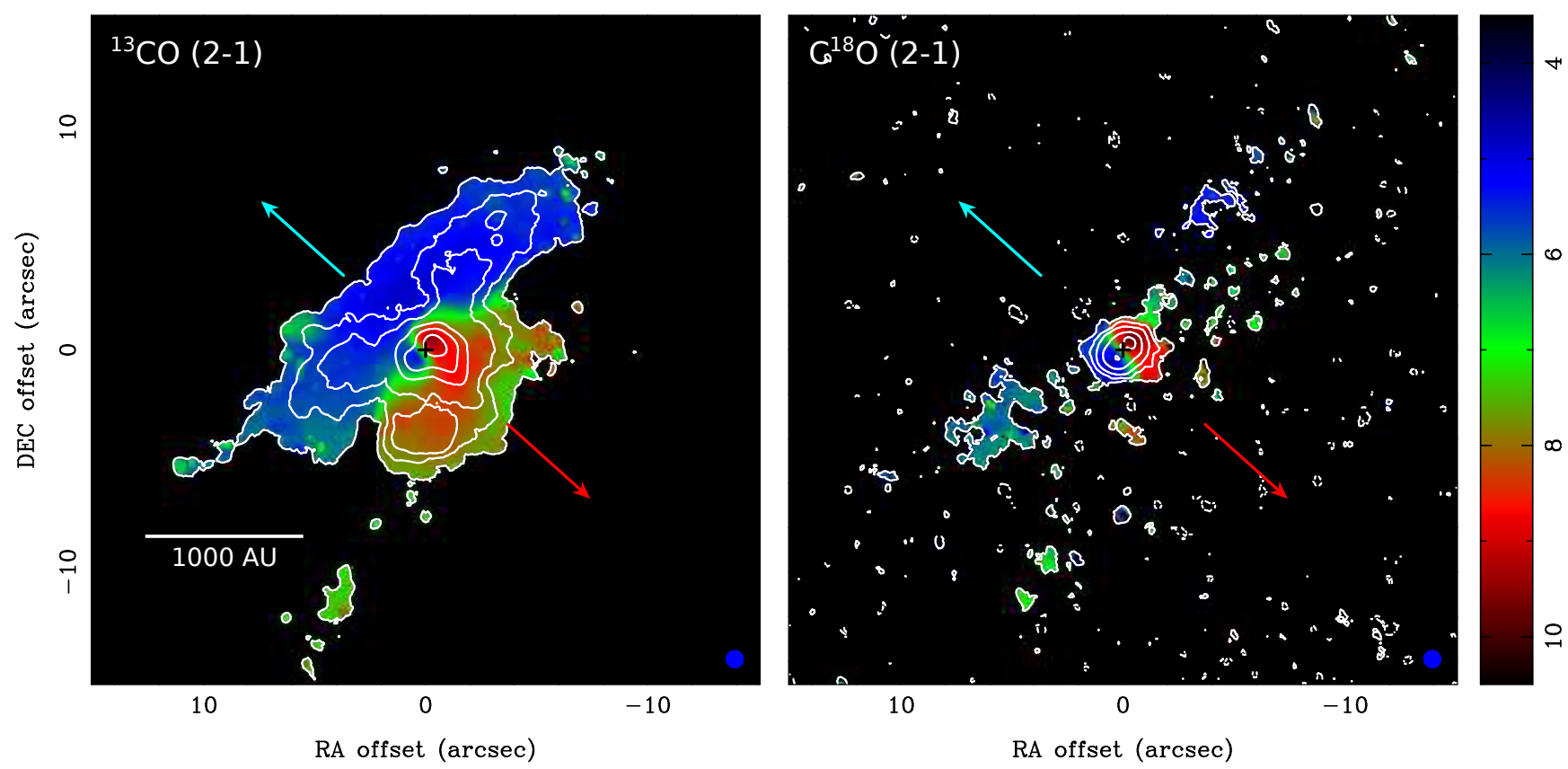

Fig. 1. Moment 0 maps (contours) overlaid on moment 1 maps (color; in units of $\mathrm{km} \mathrm{s}^{-1}$ in the LSR frame) of the ${ }^{13} \mathrm{CO}(2-1$; left $)$ and $\mathrm{C}^{18} \mathrm{O}(2-1$; right) emission in HL Tau obtained with our ALMA observations. Crosses denote the position of HL Tau. Blue and red arrows show the directions of the blue- and redshifted outflows. Blue filled ellipses present the sizes of the synthesized beams. In the ${ }^{13} \mathrm{CO}$ map, contour levels are $5 \%, 10 \%$, $15 \%, 30 \%, 50 \%$, and $80 \%$ of the peak intensity, where the $5 \%$ level corresponds to $4.5 \sigma\left(1 \sigma=13.7 \mathrm{mJy} \mathrm{Beam}^{-1} \mathrm{~km} \mathrm{~s}^{-1}\right)$. The contour levels in the $\mathrm{C}^{18} \mathrm{O}$ map are $5 \%, 15 \%, 30 \%, 60 \%$, and $90 \%$ of the peak intensity, where the $5 \%$ level corresponds to $2 \sigma\left(1 \sigma=11.6 \mathrm{mJy} \mathrm{Beam}^{-1} \mathrm{~km} \mathrm{~s}^{-1}\right)$.

and the southeast, and their elongations are perpendicular to the directions of the molecular outflows and the optical and infrared jets (Monin et al. 1996; Anglada et al. 2007; Takami et al. 2007; Movsessian et al. 2007, 2012; Hayashi \& Pyo 2009; Lumbreras \& Zapata 2014; ALMA Partnership et al. 2015; Klaassen et al. 2016). A part of the extended structures is also detected in the $\mathrm{C}^{18} \mathrm{O}$ emission, which appears as clumpy components located northwest, southeast, and south of the central component. The extended structures exhibit an overall velocity gradient in the same direction as the molecular outflows associated with HL Tau (Monin et al. 1996; Lumbreras \& Zapata 2014; ALMA Partnership et al. 2015; Klaassen et al. 2016). This velocity gradient on a 1000 au scale is also observed in the ${ }^{13} \mathrm{CO}(1-0)$ emission at lower resolutions of 3" -8 " (Sargent \& Beckwith 1987, 1991; Hayashi et al. 1993; Cabrit et al. 1996; Welch et al. 2000).

Figures 2 and 3 present the moment 0 maps of the $\mathrm{C}^{18} \mathrm{O}$ and ${ }^{13} \mathrm{CO}$ emission integrated over the four different velocity ranges, respectively. The integrated velocity ranges are high velocities $\left(V_{\mathrm{LSR}}=2.9-3.8\right.$ and $10.3-11.4 \mathrm{~km} \mathrm{~s}^{-1}$ for $\mathrm{C}^{18} \mathrm{O}$ and $V_{\mathrm{LSR}}=$ 1.9-3.8 and 10.3-12.8 $\mathrm{km} \mathrm{s}^{-1}$ for ${ }^{13} \mathrm{CO}$ ), medium-high velocities $\left(V_{\mathrm{LSR}}=3.8-4.8\right.$ and $\left.9.2-10.3 \mathrm{~km} \mathrm{~s}^{-1}\right)$, medium-low velocities $\left(V_{\mathrm{LSR}}=4.8-5.8\right.$ and $\left.8.2-9.2 \mathrm{~km} \mathrm{~s}^{-1}\right)$, and low velocities $\left(V_{\mathrm{LSR}}=\right.$ 5.8-7.1 and 7.1-8.2 $\left.\mathrm{km} \mathrm{s}^{-1}\right)$. The systemic velocity ( $\left.V_{\text {sys }}\right)$ is measured to be $V_{\mathrm{LSR}}=7.04 \mathrm{~km} \mathrm{~s}^{-1}$ (Sect. 4.1). The high-velocity blue- and redshifted $\mathrm{C}^{18} \mathrm{O}$ components are compact with sizes of $0^{\prime \prime} .3 \pm 0$. .04 (42 $\left.\pm 6 \mathrm{au}\right)$ and $0^{\prime \prime} .44 \pm 00^{\prime \prime} .02(62 \pm 3 \mathrm{au})$, and are located southeast and northwest of HL Tau with a distance of $0 \prime .5 \pm 0$ '.04 (70 $\pm 6 \mathrm{au})$, respectively. At the medium-high velocities, the sizes of the blue- and redshifted $\mathrm{C}^{18} \mathrm{O}$ components increase to 0 ".6-0 0 !9 (80-130 au), and the distance between their peak positions and HL Tau increases to $00^{\prime \prime} 6-0, \cdot 7$ (80-100 au). These results show that the $\mathrm{C}^{18} \mathrm{O}$ emission at higher velocities is located closer to HL Tau. Furthermore, the axis passing through the peak positions of the high-velocity blue- and redshifted components has a PA of $130^{\circ} \pm 3^{\circ}$, and the axis of the mediumhigh-velocity components is $133^{\circ} \pm 1^{\circ}$. Therefore, these components are well aligned along the major axis of the protoplanetary disk around HL Tau, whose PA is $138^{\circ}$ (ALMA Partnership et al. 2015). At the medium-low and low velocities, both the $\mathrm{C}^{18} \mathrm{O}$ blue- and redshifted components are elongated along the disk minor axis. In addition, the low-velocity components are centered on the position of HL Tau. Hence, the velocity structures of the central $\mathrm{C}^{18} \mathrm{O}$ emission is consistent with the observational signatures of Keplerian rotation (e.g., Dutrey et al. 1994).

In the high-velocity ${ }^{13} \mathrm{CO}$ emission (Fig. 3a), the blue- and redshifted components are compact with sizes of 0 '.3- $0^{\prime \prime} .5(50$ $70 \mathrm{au}$ ) and located close to HL Tau with a distance of $00^{\prime \prime 4}$ (60 au), similar to the high-velocity $\mathrm{C}^{18} \mathrm{O}$ emission. In addition, the axis passing through the peak positions of the high-velocity blue- and redshifted ${ }^{13} \mathrm{CO}$ components is $135^{\circ} \pm 2^{\circ}$, so they are well aligned with the disk major axis. Thus, the high-velocity ${ }^{13} \mathrm{CO}$ emission also exhibits the observational signatures of dominant rotational motion. The low-velocity blueshifted ${ }^{13} \mathrm{CO}$ emission shows an extended and elongated structure in the northwest-southeast direction with a size of $\sim 20^{\prime \prime}$ (2800 au; Fig. 3d). This extended structure is also partially detected in the $\mathrm{C}^{18} \mathrm{O}$ emission (Fig. 2d). Similar structures are also observed in the CO (1-0) emission with ALMA (ALMA Partnership et al. 2015). These structures could be related to the large-scale ambient gas. Conversely, the low-velocity redshifted ${ }^{13} \mathrm{CO}$ emission shows a bow-like structure with the apex located close to HL Tau, and its morphology is similar to the wall of the outflow cavity observed in the CO emission with SMA and ALMA (Lumbreras \& Zapata 2014; ALMA Partnership et al. 2015; Klaassen et al. 2016). Hence, the low-velocity redshifted ${ }^{13} \mathrm{CO}$ emission likely traces the cavity wall of the redshifted outflow.

At medium velocities (Figs. $3 \mathrm{~b}$ and $\mathrm{c}$ ), the ${ }^{13} \mathrm{CO}$ emission exhibits extended arc structures, as delineated by the ridges of 


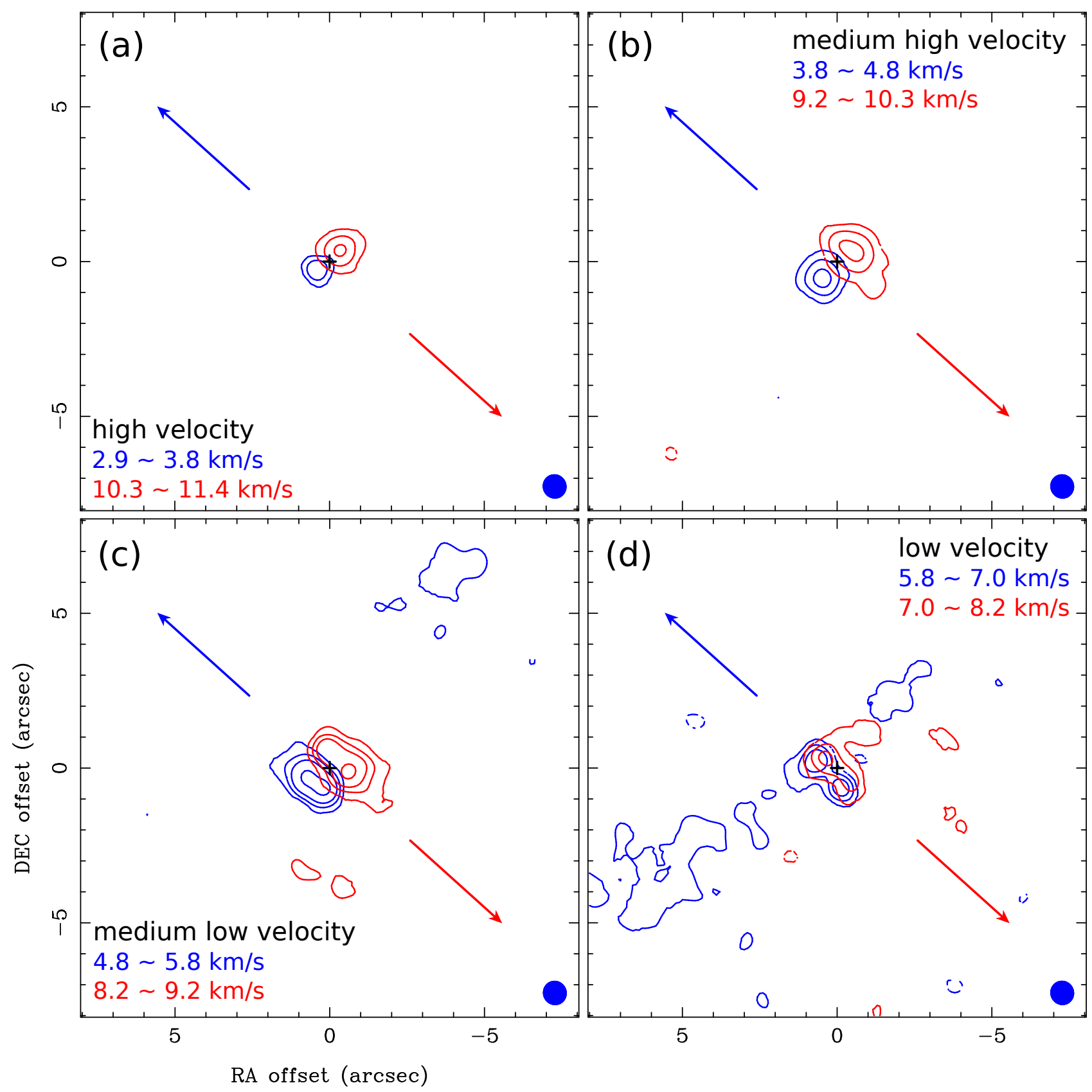

Fig. 2. Moment 0 maps of the $\mathrm{C}^{18} \mathrm{O}$ emission integrated over four different velocity ranges, high velocities $\left(V_{\mathrm{LSR}}=2.9-3.8\right.$ and $\left.10.3-11.4 \mathrm{~km} \mathrm{~s}{ }^{-1}\right)$, medium-high velocities $\left(V_{\mathrm{LSR}}=3.8-4.8\right.$ and $\left.9.2-10.3 \mathrm{~km} \mathrm{~s}^{-1}\right)$, medium-low velocities $\left(V_{\mathrm{LSR}}=4.8-5.8\right.$ and $\left.8.2-9.2 \mathrm{~km} \mathrm{~s}^{-1}\right)$, and low velocities $\left(V_{\mathrm{LSR}}=5.8-7.1\right.$ and 7.1-8.2 $\left.\mathrm{km} \mathrm{s}^{-1}\right)$. Blue and red contours present the blue- and redshifted emission, respectively. Blue and red arrows show the directions of the blue- and redshifted outflows. Crosses denote the position of HL Tau. Filled blue ellipses show the size of the synthesized beam. Contour levels are $5 \sigma, 10 \sigma$, and $15 \sigma$ in the high-velocity blueshifted map, are $5 \sigma, 15 \sigma, 30 \sigma, 50 \sigma$, and $70 \sigma$ in the high-velocity redshifted map, are $5 \sigma, 15 \sigma, 30 \sigma$, and $45 \sigma$ in the medium-high-velocity maps, are $5 \sigma, 10 \sigma, 15 \sigma, 25 \sigma, 45 \sigma$, and $75 \sigma$ in the medium-low-velocity maps, and are $5 \sigma, 10 \sigma, 15 \sigma, 25 \sigma, 45 \sigma$, and $75 \sigma$ in the low-velocity maps. The noise level in the high-velocity redshifted map is $5.1 \mathrm{mJy} \mathrm{Beam}^{-1} \mathrm{~km} \mathrm{~s}^{-1}$, and the levels in the other maps are all $3.9 \mathrm{mJy} \mathrm{Beam}^{-1} \mathrm{~km} \mathrm{~s}^{-1}$.

their contour lines (dotted curves in Fig. 3). Our method to measure the ridges of the contour lines is described in Appendix A. The entire velocity channel maps of the ${ }^{13} \mathrm{CO}$ emission in the medium-velocity ranges are shown in Appendix B. The blueand redshifted arc structures connect to the central component from the northeast and the southwest, and they stretch toward the northwest and the southeast, respectively. Figure 4 presents the spectra of the ${ }^{13} \mathrm{CO}$ emission at several positions along the arc structures. The line width of the blueshifted arc structures is $0.7 \mathrm{~km} \mathrm{~s}^{-1}$, as seen at the offsets of A. $\left(-2{ }^{\prime \prime} 8,5^{\prime \prime}\right)$ and B.(1'!9,
$3 \prime \prime 3)$. At the offsets of D. $\left(-00^{\prime \prime} 8,3^{\prime \prime}\right)$ and E. $\left(1^{\prime \prime} 2,2^{\prime \prime}\right)$, there is a secondary component at $V_{\mathrm{LSR}} \sim 5.8 \mathrm{~km} \mathrm{~s}^{-1}$ in the spectra, and it is likely associated with the large-scale ambient gas as seen in Fig. 3d. The broader line width at the offset of C.(3'?7, $\left.0^{\prime \prime} 1\right)$ is most likely due to blending of the blueshifted arc structure and the large-scale ambient gas. The spectra in the redshifted arc structure all show broader line widths of $1.4-2.4 \mathrm{~km} \mathrm{~s}^{-1}$ than those in the blueshifted arc, as seen at the offsets of F.( $\left(0^{\prime \prime} \cdot 7\right.$,

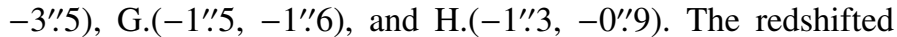
arc structure is spatially coincident with the redshifted outflow 


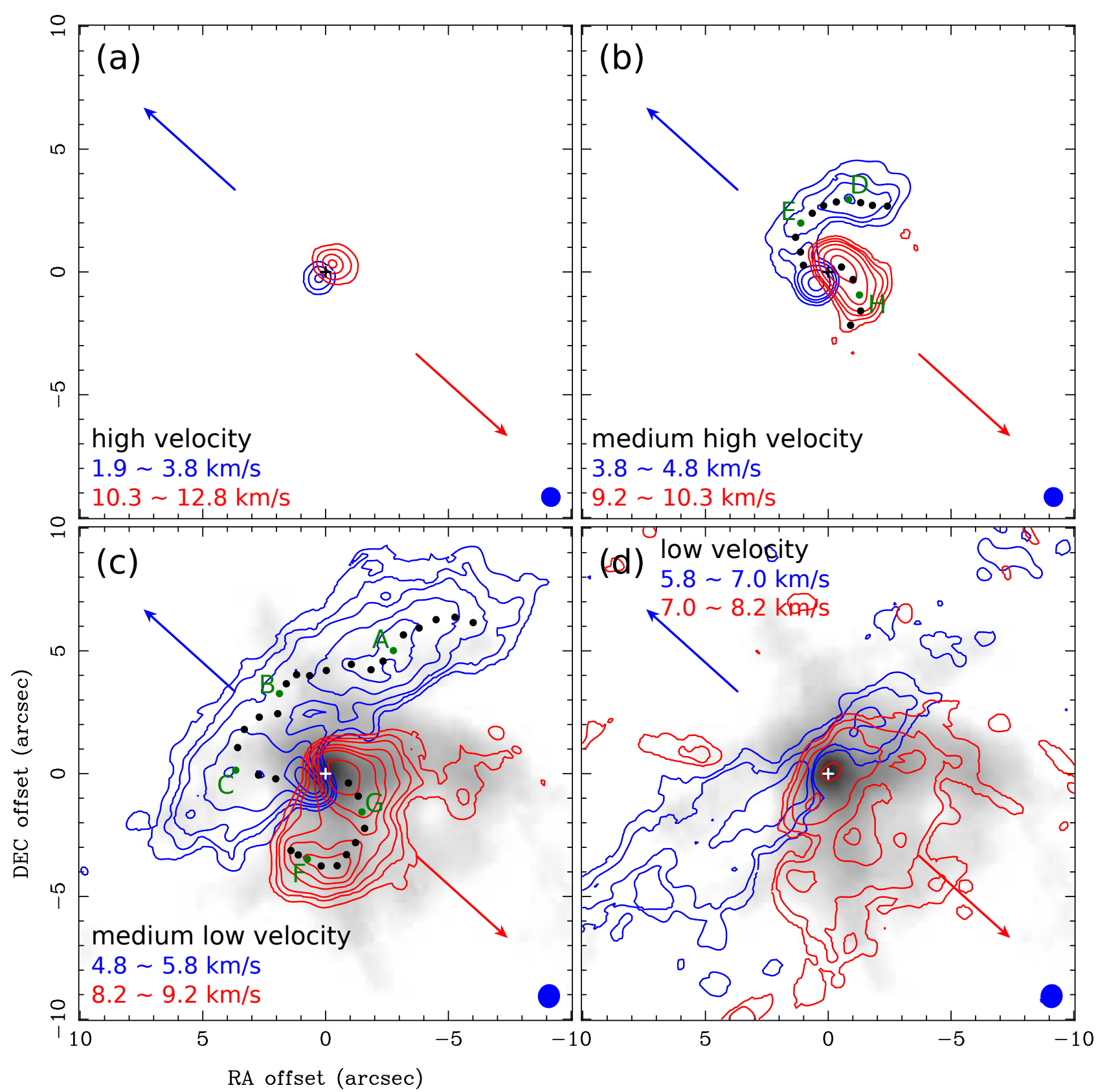

Fig. 3. Same as Fig. 2, but for the ${ }^{13} \mathrm{CO}$ emission, and the high-velocity range for the ${ }^{13} \mathrm{CO}$ emission is $V_{\mathrm{LSR}}=1.9-3.8$ and $10.3-12.8 \mathrm{~km} \mathrm{~s}{ }^{-1}$. Black and green dots delineate the ridges of the contour lines, showing the arc structures. The ridges of the contour lines were measured from the peak positions of the intensity profiles along the transverse directions of the arc structures. Green dots with labels A-H mark the positions of the extracted spectra show in Fig. 4. Gray scales in panels $c$ and $d$ present the moment 0 map of the CO (1-0) emission observed with ALMA, and the map is obtained from the data released by ALMA Partnership et al. (2015). Contour levels are $5 \sigma, 15 \sigma$, and $30 \sigma$ in the high-velocity blueshifted map, are $5 \sigma, 15 \sigma, 35 \sigma$, and $55 \sigma$ in the high-velocity redshifted map, are $5 \sigma, 15 \sigma, 25 \sigma, 45 \sigma$, and $75 \sigma$ in the medium-high-velocity maps, are $5 \sigma, 10 \sigma, 15 \sigma, 25 \sigma, 35 \sigma, 45 \sigma$, and $75 \sigma$ in the medium-low-velocity maps, and are $5 \sigma, 10 \sigma, 15 \sigma, 20 \sigma$, and $60 \sigma$ in the low-velocity maps. The noise level in the high-velocity blue- and redshifted map is 5.8 and $7.1 \mathrm{mJy} \mathrm{Beam}^{-1} \mathrm{~km} \mathrm{~s}^{-1}$, respectively, and the levels in the other maps are all $4.1 \mathrm{mJy} \mathrm{Beam}^{-1} \mathrm{~km} \mathrm{~s}^{-1}$. Significant flux is missing in the velocity ranges of panels $c$ and $d$, as shown in Figs. B.1 and B.2. The negative contours are not plotted in panels $c$ and $d$ for clarity.

observed at the low velocity (Fig. 3), and the two components are blended in the spectra. Thus, it is not straightforward to measure the line width of the redshifted arc structure. Nevertheless, the line width of the redshifted arc structure is most likely narrower than $1.4 \mathrm{~km} \mathrm{~s}^{-1}$ based on the spectrum at the offset of F. $(0$ !'7, $\left.-3^{\prime \prime} 5\right)$, where there is less contribution from the outflow.

The arc structures observed at medium-high velocities are located closer to HL Tau than those at medium-low velocities, showing that the inner part has a higher velocity than the outer part (Figs. 3b and c). Thus, there is a radial velocity gradient in each arc structure. In addition, the blue- and redshifted arc structures extend northwest and southeast, respectively. This forms an overall velocity gradient from the northwest to the southeast on a 1000 au scale. The overall velocity gradient in the northwest-southeast direction on a $1000 \mathrm{au}$ scale has been observed in the ${ }^{13} \mathrm{CO}(1-0)$ emission at lower resolutions of $3^{\prime \prime}-8^{\prime \prime}$ (Sargent \& Beckwith 1991; Hayashi et al. 1993; Cabrit et al. 1996; Welch et al. 2000). This was interpreted 

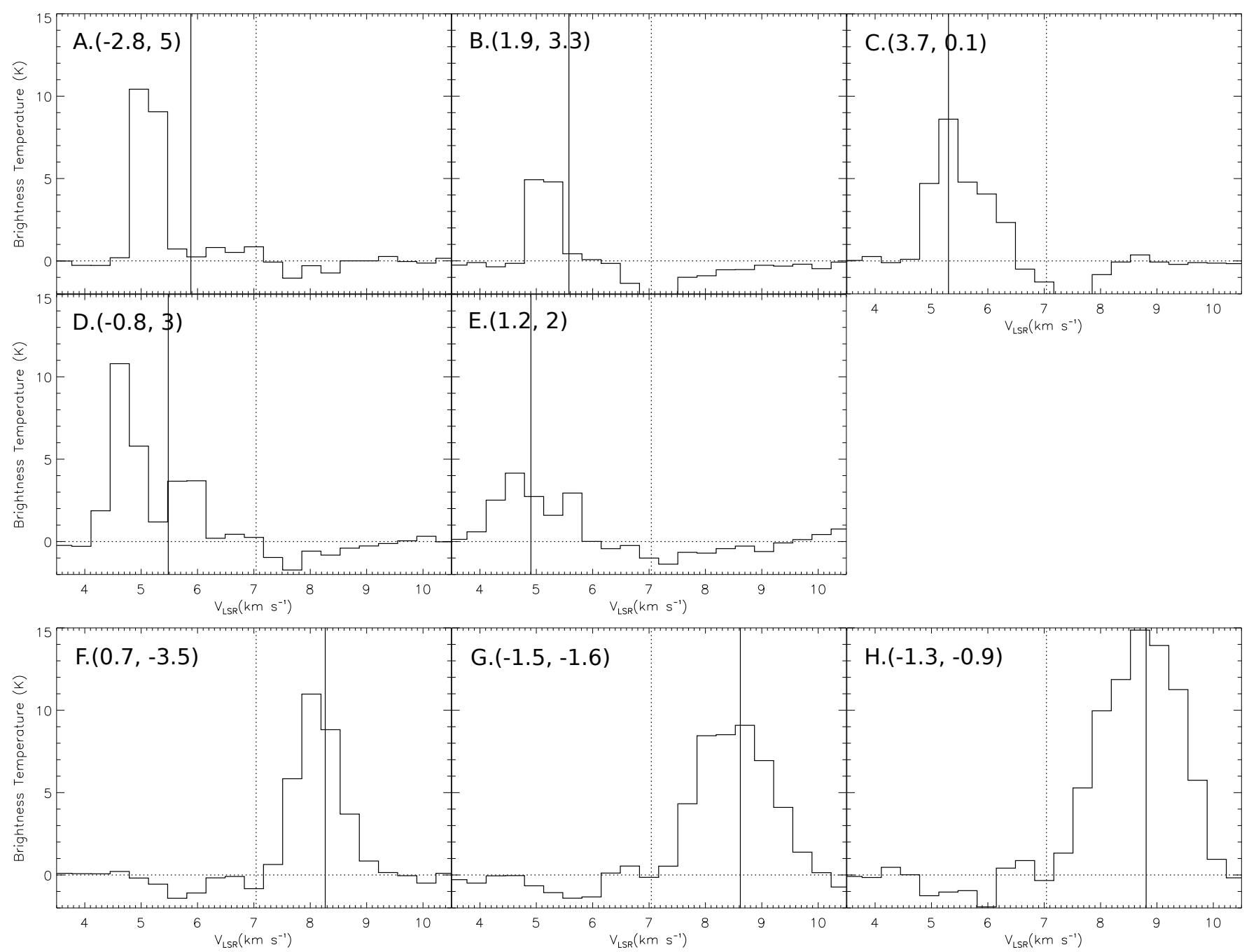

Fig. 4. Spectra of the ${ }^{13} \mathrm{CO}$ emission along the arc structures. The positions A-H, where the spectra were extracted relative to HL Tau, are labeled in the upper left corners in the panels in units of arcseconds, and they are also labeled in Fig. 3. Vertical dotted lines denote the systemic velocity measured from the disk rotation. Vertical solid lines present the expected line-of-sight velocities computed from the model of the three-dimensional infalling and rotational motions (Sect. 4.3).

as Keplerian rotation around a protostar with a mass of $0.5-$ $1 M_{\odot}$ by Sargent \& Beckwith (1991) and as sub-Keplerian rotation in a disk-like structure by Hayashi et al. (1993). However, the ALMA observations show that the direction of this velocity gradient on a 1000 au scale is opposite to that of the disk rotation on a 100 au scale, where the northwestern part is redshifted and the southeastern part is blueshifted.

\section{Analysis}

\subsection{Keplerian disk}

The $\mathrm{C}^{18} \mathrm{O}$ and ${ }^{13} \mathrm{CO}$ emission shows observational signatures of rotation. To measure the rotational profiles in the $\mathrm{C}^{18} \mathrm{O}$ and ${ }^{13} \mathrm{CO}$ emission, we followed the method described in Yen et al. (2013). We extracted position-velocity $(\mathrm{P}-\mathrm{V})$ diagrams along the disk major axis $\left(\mathrm{PA}=138^{\circ}\right)$ and measured the peak positions in given velocity channels (Figs. 5a, c, and d). The distances between the measured peak positions and HL Tau were adopted as rotational radii $\left(R_{\text {rot }}\right)$, and the velocities at the velocity channels relative to the systemic velocity $\left(\left|V_{\mathrm{LSR}}-V_{\text {sys }}\right|\right)$ were adopted as rotational velocities $\left(V_{\text {rot }}\right)$. We then fit a power-law function to these data points,

$\left|V_{\mathrm{LSR}}-V_{\text {sys }}\right|=V_{\text {rot }}\left(R_{0}\right) \times\left(\frac{R_{\text {rot }}}{R_{0}}\right)^{f} \mathrm{~km} \mathrm{~s}^{-1}$,

where $R_{0}$ is the characteristic radius and was adopted to be $1^{\prime \prime}$ $(140 \mathrm{au})$, and $f$ is the power-law index of the rotational profile. Thus, there are three free parameters in the fitting: $V_{\text {rot }}\left(R_{0}\right), f$, and $V_{\text {sys. }}$. This analysis was only applied to the $\mathrm{C}^{18} \mathrm{O}$ and ${ }^{13} \mathrm{CO}$ emission in the high-velocity ranges (Figs. 2a and 3a), where there is no contamination from the extended structures. Furthermore, there is no velocity gradient in the $\mathrm{P}-\mathrm{V}$ diagram along the disk minor axis at high velocities (Fig. 6), suggesting that the gas motion is dominated by the rotation with the least contamination from other motions in these velocity ranges. In contrast, at medium and low velocities, there is a clear velocity gradient along the disk minor axis in the $\mathrm{P}-\mathrm{V}$ diagrams of the $\mathrm{C}^{18} \mathrm{O}$ and ${ }^{13} \mathrm{CO}$ emission, which could be due to an infalling motion or outflow.

The rotational profile in the $\mathrm{C}^{18} \mathrm{O}$ emission is measured to be $(2.44 \pm 0.07) \times\left(R_{\text {rot }} / R_{0}\right)^{-0.52 \pm 0.04} \mathrm{~km} \mathrm{~s}^{-1}$ with $V_{\text {sys }}=$ $7.04 \pm 0.04 \mathrm{~km} \mathrm{~s}^{-1}$ (Fig. 5b). This rotational profile is consistent with Keplerian rotation $(f=-0.5)$ within the uncertainty. 

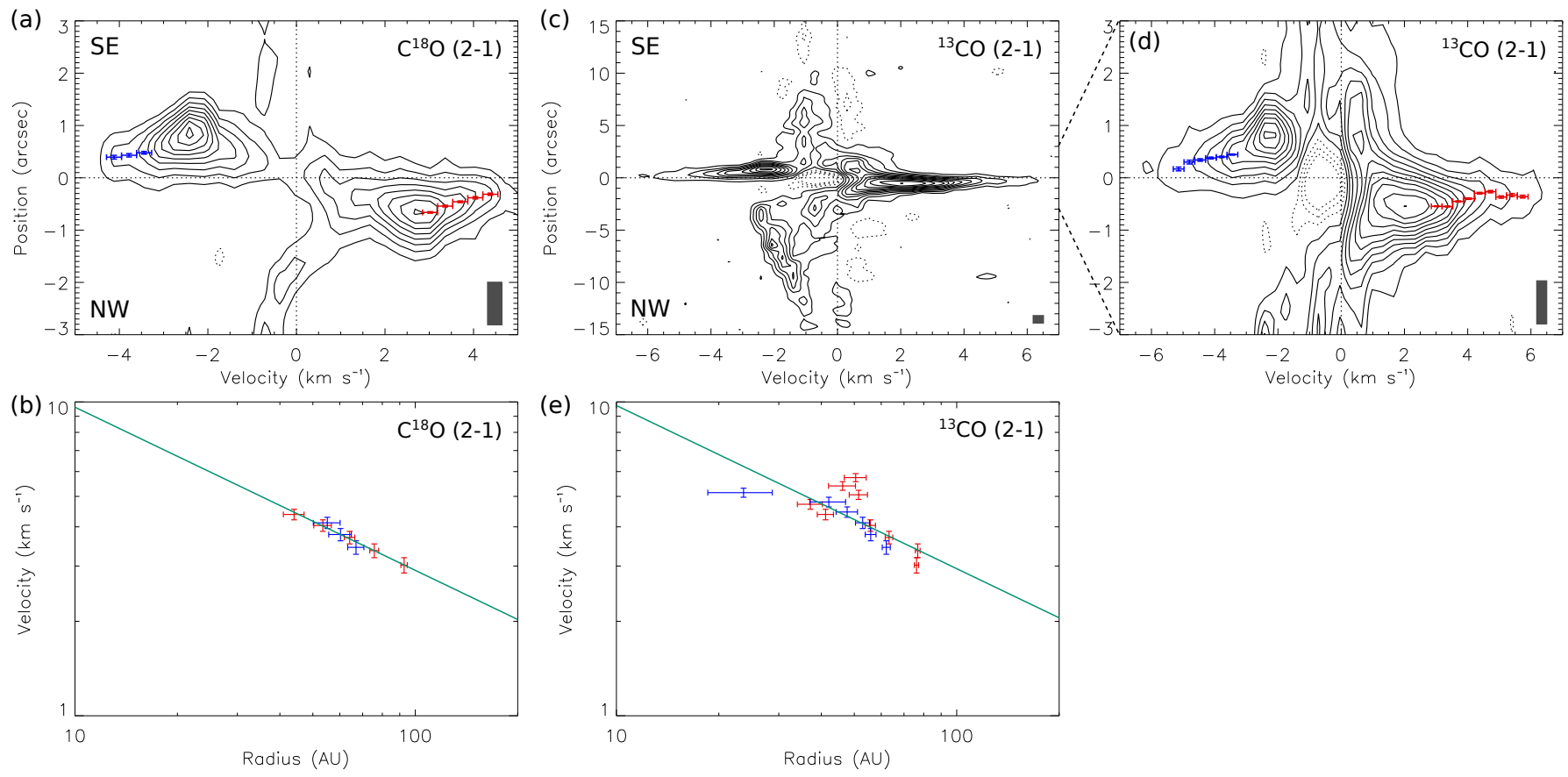

Fig. 5. Position-velocity diagrams along the disk major axis in the $\mathrm{C}^{18} \mathrm{O}$ emission (panel a) and the ${ }^{13} \mathrm{CO}$ emission (panel c). Panel $d$ : zoom-in of panel $c$. Blue and red data points show the measured peak positions in different velocity channels. Rotational profiles measured from the data points in panels $a$ and $d$ are shown in panels $b$ and $e$. Green solid lines show the best-fit power-law functions of the rotational profiles. Contour levels are from $3 \sigma$ in steps of $3 \sigma$ in panel a, where $1 \sigma$ is $6.7 \mathrm{mJy} \mathrm{Beam}^{-1}$, and the levels are from $3 \sigma$ in steps of $5 \sigma$ in panels $c$ and d, where $1 \sigma$ is $7 \mathrm{mJy} \mathrm{Beam}^{-1}$. In the P-V diagrams, vertical and horizontal dotted lines denote the systemic velocity of $V_{\mathrm{LSR}}=7.04 \mathrm{~km} \mathrm{~s}^{-1}$ and the stellar position, respectively, and gray rectangles in the bottom right corners show the angular and velocity resolutions.

Our measured $V_{\text {sys }}$ is consistent with the $V_{\text {sys }}$ estimated from the $\mathrm{CN}$ and HCN absorption observed with ALMA at a comparable angular resolution of 1" (ALMA Partnership et al. 2015). A consistent rotational profile was measured in the ${ }^{13} \mathrm{CO}$ emission, $(2.36 \pm 0.12) \times\left(R_{\text {rot }} / R_{0}\right)^{-0.56 \pm 0.06} \mathrm{~km} \mathrm{~s}^{-1}$, with $V_{\text {sys }}=$ $7.17 \pm 0.03 \mathrm{~km} \mathrm{~s}^{-1}$, although the $V_{\text {sys }}$ measured with the ${ }^{13} \mathrm{CO}$ emission is higher than the $V_{\text {sys }}$ measured with the $\mathrm{C}^{18} \mathrm{O}$ emission. When the $V_{\text {sys }}$ is fixed at $V_{\mathrm{LSR}}=7.04 \mathrm{~km} \mathrm{~s}^{-1}$, the rotational profile in the ${ }^{13} \mathrm{CO}$ emission is measured to be $(2.47 \pm 0.12) \times$ $\left(R_{\text {rot }} / R_{0}\right)^{-0.52 \pm 0.05} \mathrm{~km} \mathrm{~s}^{-1}$ (Fig. 5e). Here the rotational velocity is not yet corrected for the inclination. Our results show that the $\mathrm{C}^{18} \mathrm{O}$ and ${ }^{13} \mathrm{CO}$ emission lines in the high-velocity ranges trace the same gas motion.

The ${ }^{13} \mathrm{CO}$ emission is optically thicker than the $\mathrm{C}^{18} \mathrm{O}$ emission and is expected to trace the upper layers of the disk, where the rotational velocity is lower than in the midplane at the same radius. Nevertheless, the rotational profiles measured with these two lines are consistent within the uncertainties, suggesting that the effect of the scale height on our measurements is negligible with our sensitivity and resolutions. In addition, we note that the measured $V_{\text {sys }}$ with these two lines are different by $0.1 \mathrm{~km} \mathrm{~s}^{-1}$. The $V_{\text {sys }}$ measured with the ${ }^{13} \mathrm{CO}$ line is biased to a higher value because of the three data points at the highest redshifted velocities $\left(>5 \mathrm{~km} \mathrm{~s}^{-1}\right)$. These high-velocity data points are not detected in the $\mathrm{C}^{18} \mathrm{O}$ emission, which is likely due to the lower optical depth and thus fainter emission of the $\mathrm{C}^{18} \mathrm{O}$ line compared to the ${ }^{13} \mathrm{CO}$ line. The three data points deviate from the overall rotational profile. Their origin is not clear, and their velocity structure is not resolved, meaning that the data points at different velocities show almost consistent radii. We have tested that when we removed these three data points from the analysis, the $V_{\text {sys }}$ measured with the ${ }^{13} \mathrm{CO}$ line is consistent with that with the $\mathrm{C}^{18} \mathrm{O}$ line within the uncertainty. Nevertheless, the overall rotational profile is not affected by these data points.

From the measured rotational profiles, the protostellar mass of $\mathrm{HL}$ Tau is estimated to be $1.8 M_{\odot}$, on the assumption that the inclination angle of the protoplanetary disk is $47^{\circ}$ (ALMA Partnership et al. 2015). As discussed in Yen et al. (2013) and Aso et al. (2015), the method has a systematic error of $10-20 \%$ because of the limited resolutions and the possible contamination from other motions, so the uncertainty of our measured protostellar mass is estimated to be $0.3 M_{\odot}$. In addition, changing the inclination angle by $\pm 5^{\circ}$ results in a change in the measured protostellar mass by ${ }_{-0.2}^{+0.4} M_{\odot}$. Our measured protostellar mass is consistent with $1.7 M_{\odot}$ estimated from the P-V diagrams in the $\mathrm{HCO}^{+}(1-0)$ and $\mathrm{CO}(1-0)$ emission observed with ALMA at a comparable angular resolution of $\sim 1^{\prime \prime}$ by Pinte et al. (2016).

To estimate the disk mass, we fit a two-dimensional Gaussian function to the central $\mathrm{C}^{18} \mathrm{O}$ emission in the moment 0 map (Fig. 1). The total flux is measured to be $2.26 \pm 0.22 \mathrm{Jy} \mathrm{km} \mathrm{s}-1$ with the deconvolved FWHM size of $1^{\prime \prime} .87 \pm 0$ '. $12 \times 1^{\prime \prime} .28 \pm 0$ "..08 $(262 \pm 17$ au $\times 179 \pm 11 \mathrm{au})$. The gas kinematic temperature in the protoplanetary disk around HL Tau was estimated to be $60 \mathrm{~K}$ at the outer radii of 60-100 au from the ALMA observations in the CO (1-0) emission (Yen et al. 2016). On the assumption of a local thermal equilibrium (LTE) condition and optically thin $\mathrm{C}^{18} \mathrm{O}$ emission, the mass traced by the $\mathrm{C}^{18} \mathrm{O}$ emission is estimated to be $3.5 \times 10^{-3} M_{\odot}$ with an excitation temperature of $60 \mathrm{~K}$ and a $\mathrm{C}^{18} \mathrm{O}$ abundance of $1.5 \times 10^{-7}$ relative to $\mathrm{H}_{2}$ (Brittain et al. 2005; Smith et al. 2015). When the excitation temperature is assumed to be $100 \mathrm{~K}$ (Brittain et al. 2005), the estimated mass becomes $5 \times 10^{-3} M_{\odot}$. The gas mass in the protoplanetary disk around HL Tau estimated with the $\mathrm{C}^{18} \mathrm{O}$ emission is $0.2-0.3 \%$ of the protostellar mass and is comparable 

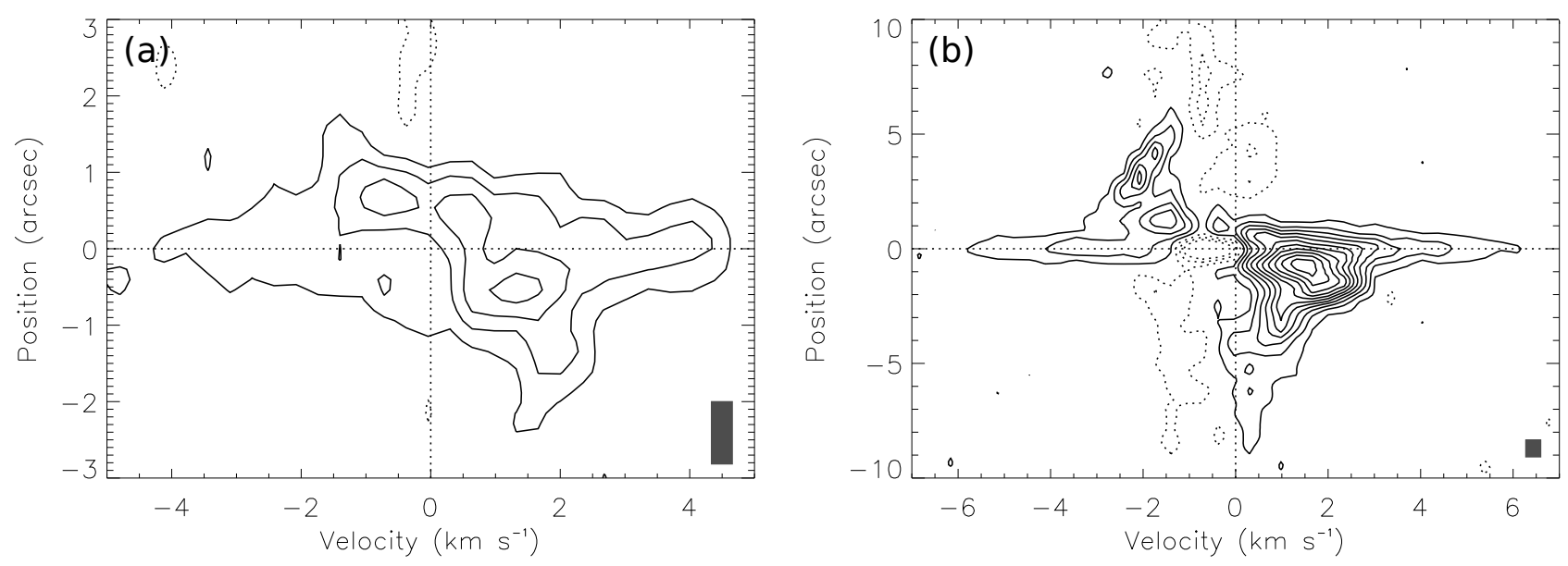

Fig. 6. Position-velocity diagrams along the disk minor axis in the $\mathrm{C}^{18} \mathrm{O}$ emission (panel $a$ ) and the ${ }^{13} \mathrm{CO}$ emission $($ panel $b$ ). Contour levels are from $3 \sigma$ in steps of $3 \sigma$ in panel $a$ and are from $3 \sigma$ in steps of $5 \sigma$ in panel $b$. Gray rectangles in the bottom right corners in the $\mathrm{P}-\mathrm{V}$ diagrams show the angular and velocity resolutions.

to the dust mass estimated with the continuum emission, $(0.3-$ 3) $\times 10^{-3} M_{\odot}$ (D'Alessio et al. 1997; Men'shchikov et al. 1999; Kwon et al. 2011; Pinte et al. 2016; Carrasco-González et al. 2016). This could imply a low gas-to-dust mass ratio of $<10$. Such a low gas-to-dust mass ratio in the protoplanetary disk around HL Tau has been suggested by Pinte et al. (2016) by modeling the $2.9 \mathrm{~mm}, 1.3 \mathrm{~mm}$, and $0.87 \mathrm{~mm}$ continuum data obtained with ALMA. On the other hand, observations have found that $\mathrm{CO}$ molecules can be depleted by two orders of magnitude in protoplanetary disks because of carbon depletion (e.g., Schwarz et al. 2016). If that is the case, the $\mathrm{C}^{18} \mathrm{O}$ abundance can be lower than the typical value in the ISM even when the temperature is higher than the $\mathrm{CO}$ sublimation temperature, and the disk mass estimated with the $\mathrm{C}^{18} \mathrm{O}$ emission in HL Tau would be higher, resulting in a higher gas-to-dust mass ratio. Moreover, the radiative transfer model suggests that the $1.3 \mathrm{~mm}$ continuum emission in the protoplanetary disk around HL Tau is optically thick, with $\tau$ ranging from 2 to 6 , except at the radii of the gaps (Pinte et al. 2016). The gas mass of the disk estimated from our continuum-subtracted $\mathrm{C}^{18} \mathrm{O}$ flux can be biased to a lower value because of the continuum optical depth. Thus, our estimated disk gas mass should be considered as a lower limit because of the possible $\mathrm{CO}$ depletion and the effect of the continuum opacity.

\subsection{Temperature, column density, and mass of the arc structures}

In the medium-low velocity range, the $\mathrm{C}^{18} \mathrm{O}$ emission exhibits a blueshifted component located at $\sim 7^{\prime \prime}$ northwest of HL Tau (Fig. 2c). This $\mathrm{C}^{18} \mathrm{O}$ component is coincident with the northwestern intensity peak in the blueshifted arc structure in the ${ }^{13} \mathrm{CO}$ emission in the same velocity range (Fig. 3). On the assumption that this $\mathrm{C}^{18} \mathrm{O}$ component and the coincident ${ }^{13} \mathrm{CO}$ emission trace the same bulk of gas, we estimated the gas kinematic temperature and column density by comparing the $\mathrm{C}^{18} \mathrm{O}$ and ${ }^{13} \mathrm{CO}$ brightness temperatures. Figure 7 presents the spectra of the $\mathrm{C}^{18} \mathrm{O}$ and ${ }^{13} \mathrm{CO}$ emission averaged over the region within a radius of $0^{\prime \prime} .35$ centered on the northwestern $\mathrm{C}^{18} \mathrm{O}$ component. The averaged area is the same as the size of the synthesized beam. The spectra show that the $\mathrm{C}^{18} \mathrm{O}$ and ${ }^{13} \mathrm{CO}$ emission lines are both detected in two velocity channels, corresponding to a line width of $0.68 \mathrm{~km} \mathrm{~s}^{-1}$. The mean brightness temperatures of the $\mathrm{C}^{18} \mathrm{O}$ and ${ }^{13} \mathrm{CO}$ emission over the two velocity channels are

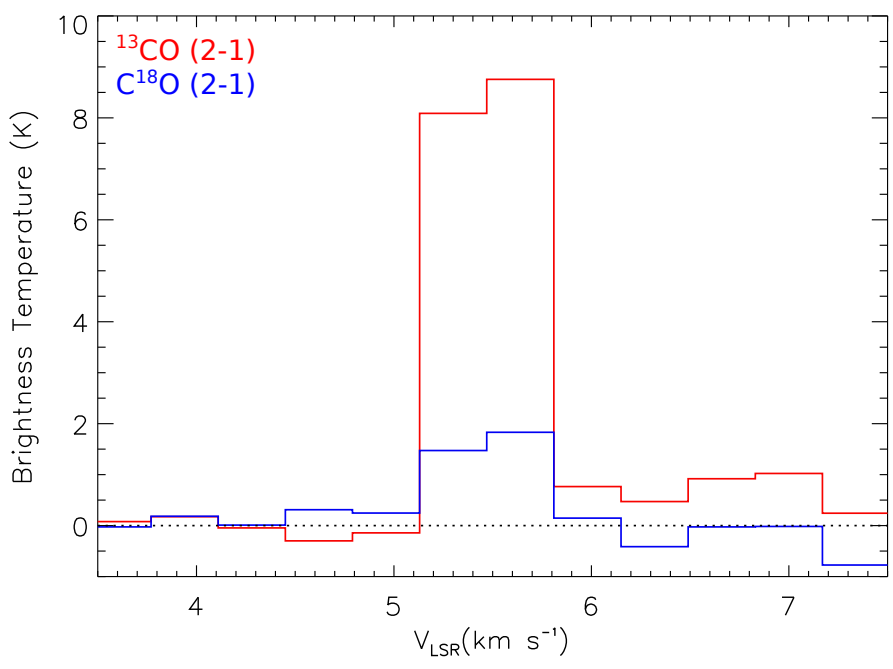

Fig. 7. Spectra of the $\mathrm{C}^{18} \mathrm{O}$ (blue histogram) and ${ }^{13} \mathrm{CO}$ (red histogram) emission averaged over the area within a radius of $0^{\prime \prime} .35$ centered on the offset of $\left(-3^{\prime \prime} 5,6\right.$, 5$)$, that is, the northwestern $\mathrm{C}^{18} \mathrm{O}$ component at the medium-low velocity in Fig. 2c. The averaged area corresponds to the area of the synthesized beam.

measured to be $T_{\mathrm{C}^{18} \mathrm{O}}=1.7 \pm 0.2 \mathrm{~K}$ and $T_{{ }^{13} \mathrm{CO}}=8.4 \pm 0.2 \mathrm{~K}$, respectively. The $\mathrm{C}^{18} \mathrm{O}(2-1)$ and ${ }^{13} \mathrm{CO}(2-1)$ lines are thermalized at typical densities in protostellar sources on a 1000 au scale, where $n\left(\mathrm{H}_{2}\right)>10^{4} \mathrm{~cm}^{-3}$ (e.g., Shirley et al. 2000). Thus, the excitation temperatures of these two lines are expected to be the same as the gas kinematic temperature. The excitation temperature $\left(T_{\mathrm{ex}}\right)$ and their optical depths $\left(\tau_{\mathrm{C}^{18} \mathrm{O}}\right.$ and $\left.\tau_{{ }^{13} \mathrm{CO}}\right)$ can be derived from their line ratio as

$\frac{T_{{ }^{13} \mathrm{CO}}}{T_{\mathrm{C}^{18} \mathrm{O}}}=\left(\frac{B_{v_{\mathrm{C}^{18} \mathrm{O}}}\left(T_{\mathrm{ex}}\right)-B_{v_{\mathrm{C}^{18} \mathrm{O}}}\left(T_{\mathrm{bg}}\right)}{B_{v_{13} \mathrm{CO}}\left(T_{\mathrm{ex}}\right)-B_{v_{13} \mathrm{CO}}\left(T_{\mathrm{bg}}\right)}\right)\left(\frac{1-e^{-\tau_{\mathrm{C}}{ }^{18} \mathrm{O}}}{1-e^{-\tau_{13} \mathrm{CO}}}\right)$,

where $B_{v}$ is the Planck function at the frequency $v, v_{\mathrm{C}^{18} \mathrm{O}}$ and $v_{13} \mathrm{CO}$ are the rest frequencies of $\mathrm{C}^{18} \mathrm{O}(2-1)$ and ${ }^{13} \mathrm{CO}(2-1)$, and $T_{\mathrm{bg}}$ is the cosmic background temperature of $2.73 \mathrm{~K}$. The ratio of $\tau_{\mathrm{C}^{18} \mathrm{O}}$ to $\tau_{{ }^{13} \mathrm{CO}}$ can be computed with a given $T_{\mathrm{ex}}$ and a ${ }^{13} \mathrm{CO} / \mathrm{C}^{18} \mathrm{O}$ abundance ratio to solve the above equation. In our calculation, the ${ }^{13} \mathrm{CO} / \mathrm{C}^{18} \mathrm{O}$ abundance ratio was adopted to be 10 , which was measured from infrared spectra of $\mathrm{CO}$ and its isotopologues in HL Tau (Brittain et al. 2005; Smith et al. 2015). This ${ }^{13} \mathrm{CO} / \mathrm{C}^{18} \mathrm{O}$ 
abundance ratio is higher than the typical ratio of 7 in the ISM and 5.5 in the solar system (Wilson \& Rood 1994). With Eq. (2), $T_{\mathrm{ex}}$ is derived to be $15 \mathrm{~K}, \tau_{\mathrm{C}^{18} \mathrm{O}}$ to be 0.18 , and $\tau_{{ }^{13} \mathrm{CO}}$ to be 1.78. Thus, the gas kinematic temperature at the northwestern peak in the arc structures is estimated to be $15 \mathrm{~K}$. Assuming this component is in the disk plane, its deprojected radius is 7"!6 (1060 au). The estimated gas kinematic temperature is consistent with the expected dust temperature at a distance of $1000 \mathrm{au}$ from a star with a luminosity of 7.6 $L_{\odot}(15 \mathrm{~K}$; Beckwith et al. 1990; Hayashi et al. 1993), and it is lower than the gas temperature of $\sim 60 \mathrm{~K}$ in the outer region ( $\sim 60-100 \mathrm{AU})$ of the protoplanetary disk around HL Tau measured with the ALMA CO (1-0) observations (Yen et al. 2016). When a lower ${ }^{13} \mathrm{CO} / \mathrm{C}^{18} \mathrm{O}$ abundance ratio of 5.5 is adopted, the derived $T_{\mathrm{ex}}, \tau_{\mathrm{C}^{18} \mathrm{O}}$, and $\tau_{{ }^{13} \mathrm{CO}}$ become $38 \mathrm{~K}, 0.05$, and 0.3 , respectively.

Except for the northwestern $\mathrm{C}^{18} \mathrm{O}$ component, no other $\mathrm{C}^{18} \mathrm{O}$ counterpart of the arc structures is clearly detected. Thus, to estimate the mass of the arc structures, we interpolated the temperature assuming it is a power-law profile, and the temperature is $15 \mathrm{~K}$ at a radius of $1000 \mathrm{au}$ (this work) and $60 \mathrm{~K}$ at a radius of $100 \mathrm{au}$ (Yen et al. 2016). The power-law index is derived to be -0.6 . Then, we computed the optical depth of the ${ }^{13} \mathrm{CO}$ emission pixel by pixel from its mean brightness temperature in the medium-velocity ranges on the assumption of a LTE condition. Our calculation shows that the ${ }^{13} \mathrm{CO}$ emission is mostly optically thin to close to optically thick, with $\tau$ ranging from 0.3 to 1 , except for the northwestern end of the blueshifted arc structure, where $\tau$ increases to $2-3$. From Figs. 3b and c, the integrated fluxes of the blue- and redshifted arc structures are measured to be $866 \mathrm{Jy} \mathrm{km} \mathrm{s}^{-1}$ over an area of 194 square arcsecond and $533 \mathrm{Jy} \mathrm{km} \mathrm{s}^{-1}$ over an area of 110 square arcsecond, respectively. To exclude the contribution from the protoplanetary disk at medium velocities, the flux in the central region, where intense $\mathrm{C}^{18} \mathrm{O}$ emission is present (light blue contours in Fig. 8), was not integrated. The masses of the blueand redshifted arc structures are estimated to be $2.9 \times 10^{-3} M_{\odot}$ and $2.8 \times 10^{-3} M_{\odot}$ on the assumption of the ${ }^{13} \mathrm{CO}$ abundance of $1.5 \times 10^{-6}$ relative to $\mathrm{H}_{2}$ (Brittain et al. 2005; Smith et al. $2015)$. The correction of the optical depth, $\tau /\left(1-\exp ^{-\tau}\right)$, was performed pixel by pixel. The estimated mass of the arc structures is comparable to or lower than the mass of the protoplanetary disk estimated from the $\mathrm{C}^{18} \mathrm{O}$ emission (Sect. 4.1) and also from the continuum emission (0.003-0.3 $M_{\odot}$; e.g., Pinte et al. 2016; Carrasco-González et al. 2016). In addition, it is approximately one-tenth of the total mass on a scale of $20^{\prime \prime}$ (2800 au) around HL Tau measured with the Nobeyama Millimeter Array at an angular resolution of $5^{\prime \prime}\left(0.03 M_{\odot}\right.$; Hayashi et al. 1993) and with the IRAM $30 \mathrm{~m}$ telescope at an angular resolution of $22^{\prime \prime}$ (0.06 $M_{\odot}$; Cabrit et al. 1996). The interpolated temperature profile shows that the kinematic temperature is higher than $20 \mathrm{~K}$, the $\mathrm{CO}$ sublimation temperature, at a radius smaller than $600 \mathrm{au}$. Hence, our mass estimation of the arc structures is likely not affected by $\mathrm{CO}$ frozen out onto dust grains, except for the northwestern end of the blueshifted arc structure, where the temperature is likely lower than $20 \mathrm{~K}$.

\subsection{Kinematics of arc structures}

Figure 8 presents the moment 0 maps of the ${ }^{13} \mathrm{CO}$ emission integrated over the medium-velocity ranges only and overlaid on the moment 1 map of the ${ }^{13} \mathrm{CO}$ emission computed with the entire velocity range to emphasize the velocity pattern of the arc structures. The light blue contours show the moment 0 map of the $\mathrm{C}^{18} \mathrm{O}$ emission. The velocity features inside these $\mathrm{C}^{18} \mathrm{O}$ contours are dominated by the disk component, and they are therefore not discussed here. The arc structures exhibit a dominant velocity gradient along the disk minor axis from the northeast (blueshifted) to the southwest (redshifted). In addition, there is a smaller velocity gradient along the disk major axis, where the northwestern part is more blueshifted than the southeastern part. This is more clearly shown in the $\mathrm{P}-\mathrm{V}$ digram along the major axis (Fig. 5c). The southeastern part is centered on the relative velocity of $-1 \mathrm{~km} \mathrm{~s}^{-1}$, and the northwestern part is distributed at the relative velocity from $-2.5 \mathrm{~km} \mathrm{~s}^{-1}$ to $-1 \mathrm{~km} \mathrm{~s}^{-1}$ with respect to $V_{\text {sys }}=-7 \mathrm{~km} \mathrm{~s}^{-1}$. To study these observed velocity structures of the arc structures, we computed model moment 1 maps of different types of gas motions and compared them with the observations.

\subsubsection{Keplerian rotation model}

Figure $8 \mathrm{~b}$ presents the model moment 1 map assuming that the arc structures follow Keplerian rotation around HL Tau with a protostellar mass of $1.8 M_{\odot}$. We assume that the arc structures are in the disk plane with an inclination angle of $47^{\circ}$ and a PA of $138^{\circ}$, the same as the central protoplanetary disk. The model moment 1 map shows a clear velocity gradient along the disk major axis, where the northwestern part is redshifted and the southeastern part is blueshifted, and there is no velocity gradient along the disk minor axis. In our observations, the northwestern part of the arc structures is blueshifted, and the velocity pattern is not symmetric with respect to the disk minor axis. In addition, a velocity gradient along the disk minor axis is clearly observed. These features are inconsistent with the expectation from a dominant rotational motion, and thus the motion of the arc structures is not pure rotation around HL Tau.

\subsubsection{Infall and rotation model}

The arc structures exhibit a clear velocity gradient along the disk minor axis (northeast-southwest direction), which was interpreted as infalling and rotational motions in a disk-like structure by Hayashi et al. (1993). To compare our observations with this interpretation, we computed models of an infalling and rotating geometrically thin envelope with our measured protostellar mass and specific angular momentum (see Appendix C.1). The moment 1 map of this model shows a clear velocity gradient along the disk minor axis similar to the observations (Fig. 8c), suggesting that the observed velocity gradient along the disk minor axis can be caused by infalling and rotational motions. However, in the model moment 1 map, the velocity in the northwestern region is close to the systemic velocity because the velocity of the infalling motion in the disk plane projected onto the line of sight approaches zero near the disk major axis. In addition, the rotational motion induces redshifted emission in the northwest, as demonstrated in Fig. 8b. As a result, there is no blueshifted velocity in the northwest in the model moment 1 map. This is inconsistent with the observations that show clear blueshifted emission in the northwest. The infalling and rotational motions in the disk plane therefore cannot fully explain the gas motions of the arc structures.

\subsubsection{Infall and counter-rotation model}

Alternatively, when the arc structures are infalling and rotating in a counter direction with respect to the disk rotation, this counter rotation can induce blueshifted emission toward the northwest 

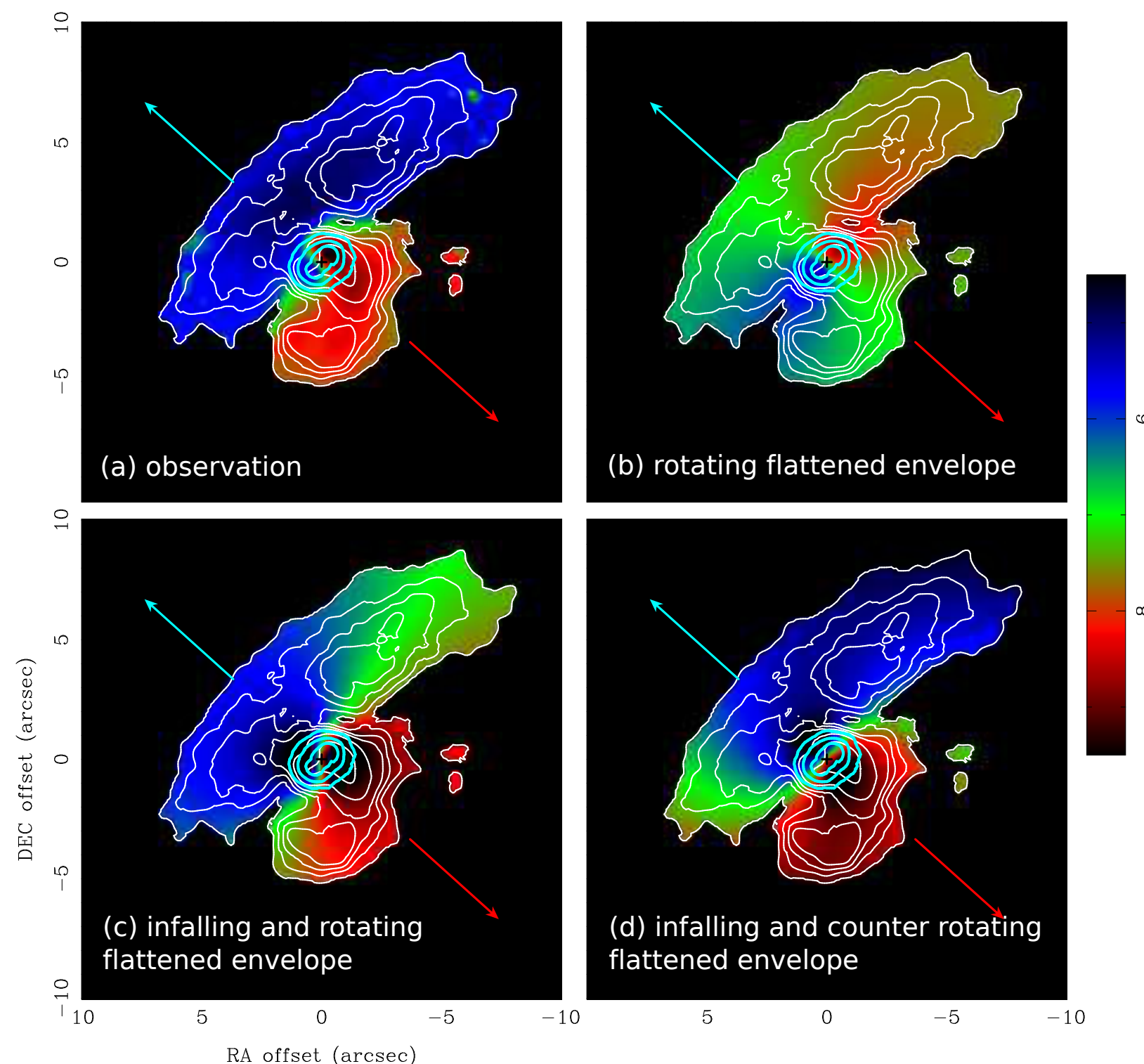

(b) rotating flattened envelope

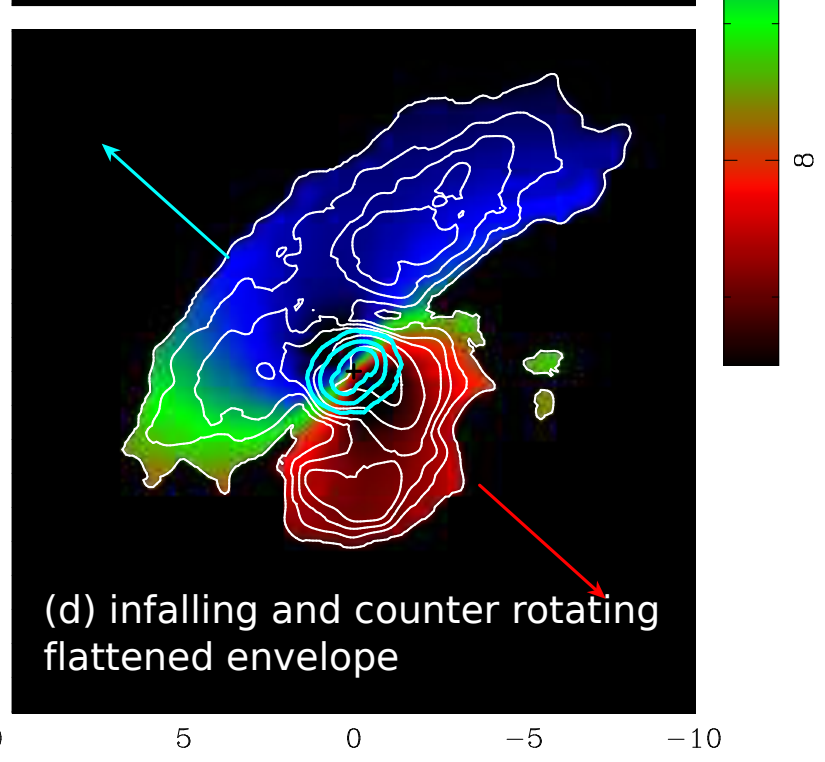

Fig. 8. White and light blue contours present the moment 0 map of the ${ }^{13} \mathrm{CO}$ emission at the medium velocities and the $\mathrm{C}^{18} \mathrm{O}$ emission, respectively. Color scales present the moment 1 maps of panel $a$ : the observed ${ }^{13} \mathrm{CO}$ emission computed with the entire velocity range, same as Fig. 1 left; panel $b$ : Keplerian rotating envelope model; panel $c$ : infalling and rotating envelope model; and panel $d$ : infalling and counter-rotating envelope model in units of $\mathrm{km} \mathrm{s}^{-1}$ in the LSR frame. Blue and red arrows show the directions of the blue- and redshifted outflows. Crosses denote the position of HL Tau. Contour levels are 5\%, 10\%, 15\%, 20\%, 35\%, 70\%, and 90\% of the peak intensity in the ${ }^{13} \mathrm{CO}$ map, and are $20 \%$, 50\%, and $80 \%$ of the peak intensity in the $\mathrm{C}^{18} \mathrm{O}$ map. The peak intensities are 0.78 and $0.41 \mathrm{Jy} \mathrm{Beam}^{-1} \mathrm{~km} \mathrm{~s}^{-1}$, corresponding to $94 \sigma$ and $52 \sigma$, in the ${ }^{13} \mathrm{CO}$ and $\mathrm{C}^{18} \mathrm{O}$ maps, respectively.

and redshifted emission toward the southeast. To compare this scenario with the observations, we computed models of an infalling and counter-rotating geometrically thin envelope (see Appendix C.2). The moment 1 map of this model shows that the counter rotation indeed induces blueshifted emission in the northwest, similar to the observations (Fig. 8d). However, the velocity of the eastern part of the arc structures in the model moment 1 map is close to the systemic velocity and even becomes redshifted. The reason is that the counter-rotation induces redshifted emission in the southeast and compensates for blueshifted emission induced by the infalling motion. This feature is different from the observations, where the eastern part of the arc structures is clearly blueshifted. Therefore, the gas motions of the arc structures cannot be fully explained either with our model of the infalling and counter-rotational motions following a simple rotational profile.

\subsubsection{Three-dimensional infall and rotation model}

We then considered three-dimensional infalling motion because infalling gas above the disk plane can have a non-zero line-ofsight velocity $\left(V_{\text {los }}\right)$ along the disk major axis after projection, different from infalling motion with the geometrically thin approximation. In this model, we adopted the velocity profiles of three-dimensional infalling and rotational motions from Ulrich (1976) and Mendoza et al. (2004). To take all the possible orientations of the arc structures in the three-dimensional space into account, we assumed that the arc structures are composed of an assemble of infalling and rotating particles, and each particle can be located anywhere along the line of sight. In this case, all the possible angles between the velocity vectors and a given line of sight were computed. For each pixel, we searched for the model 

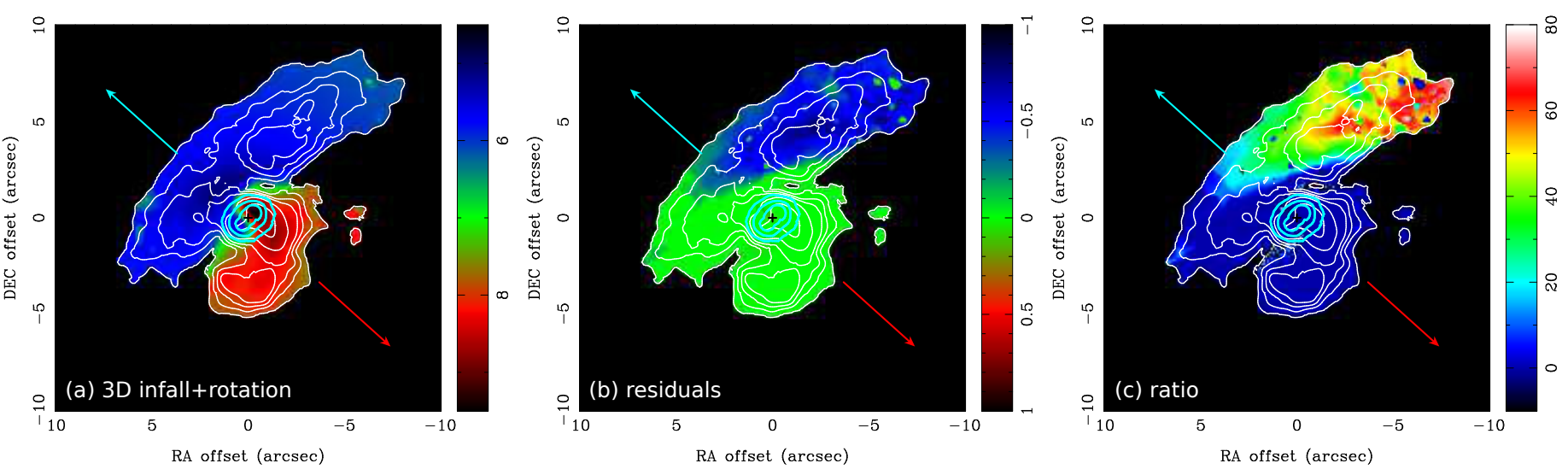

Fig. 9. Panel $a$ : model moment 1 map (color) that best matches the observations, assuming the gas kinematics traced by the ${ }^{13} \mathrm{CO}$ emission is the three-dimensional infalling and rotational motions as described by Eqs. (C.4)-(C.7). Panel b: residual moment 1 map (color) after subtracting the model moment 1 map, panel $a$ from the observed moment 1 map, Fig. 8a. Panel $c$ : ratio of the residuals to the expected relative velocity from the model. The color scale shows the percentages. White and light blue contours present the observed moment 0 maps of the ${ }^{13} \mathrm{CO}$ emission at the medium velocities and the $\mathrm{C}^{18} \mathrm{O}$ emission, the same as those in Fig. 8. Blue and red arrows show the directions of the blue- and redshifted outflows. Crosses denote the position of HL Tau.

$V_{\mathrm{LSR}}$ that best matched the observations. The details of the model calculations are described in Appendix C.3.

The model moment 1 map indeed shows similar velocity features to the observations, suggesting that the kinematics of the arc structures can be explained with the three-dimensional infalling and rotational motions, where the blue- and redshifted arc structures are located behind and in front of the disk plane, respectively. Figure $9 \mathrm{~b}$ presents the residuals after subtracting the model from the observed moment 1 map. The residual map shows that the observed velocity of the redshifted arc structure and the inner and eastern parts of the blueshifted arc structure can be reproduced with the model. However, the observed velocity in the northwestern part of the blueshifted arc structure is clearly more strongly blueshifted than the model. The blueshifted velocity excess is as high as $0.8 \mathrm{~km} \mathrm{~s}^{-1}$, significantly higher than the velocity resolution of $0.34 \mathrm{~km} \mathrm{~s}^{-1}$. In other words, the northwestern part of the blueshifted arc structure has a relative velocity higher than the expected free-fall velocity. We note that this velocity excess is the difference between the observed and model $V_{\mathrm{LSR}}$ and is not yet corrected for the projection. Figure $9 \mathrm{c}$ shows the ratio of the residuals to the expected relative velocity (i.e., $V_{\text {los }}$ in Eq. (C.7)) from the model. Considering the projection, the observed velocity excess is estimated to be $\sim 60-70 \%$ of the expected free-fall velocity, which is significantly higher than the uncertainty in the protostellar mass. As all the possible angles between the velocity vector and the line of sight were considered in our model calculation, the deviation between the observed velocity and the expected free-fall velocity is not due to the orientation of the arc structures in the three-dimensional space. This result suggests that the gravity of HL Tau is insufficient to accelerate the infalling gas to the observed velocity. Therefore, the kinematics of the whole arc structures cannot simply be interpreted as infalling flows toward the disk with the conventional model.

\subsubsection{Effects of missing flux on the observed velocity}

We note that our ALMA observations suffer from missing flux, which may bias our observed mean velocity. HL Tau has been observed with the IRAM $30 \mathrm{~m}$ telescope in the ${ }^{13} \mathrm{CO}(2-1)$ and (1-0) emission (Monin et al. 1996; Cabrit et al. 1996). The systemic velocity of the large-scale ambient gas on a scale of $2^{\prime}(17000 \mathrm{au})$ around HL Tau is estimated to be $V_{\mathrm{LSR}}=6.3-$ $6.6 \mathrm{~km} \mathrm{~s}^{-1}$, which is $0.4-0.7 \mathrm{~km} \mathrm{~s}^{-1}$ more blueshifted than the systemic velocity of HL Tau estimated from the disk rotation. We convolved our ${ }^{13} \mathrm{CO}$ image with the angular resolution of the IRAM 30 m observations in the ${ }^{13} \mathrm{CO}(2-1)$ emission, $11^{\prime \prime} 4$, and compared the observed brightness temperature at the position of HL Tau. In the blueshifted velocity range, the missing flux is $\sim 40 \%$ at $V_{\mathrm{LSR}}=4.6 \mathrm{~km} \mathrm{~s}^{-1}$ and increases to $80 \%$ at $V_{\mathrm{LSR}}=5.6 \mathrm{~km} \mathrm{~s}^{-1}$. The missing flux is the highest and more than $95 \%$ at the systemic velocity of the ambient gas, $V_{\mathrm{LSR}}$ $=6.3-6.5 \mathrm{~km} \mathrm{~s}^{-1}$. At the systemic velocity of the protoplanetary disk around HL Tau, $V_{\mathrm{LSR}}=7 \mathrm{~km} \mathrm{~s}^{-1}$, the missing flux is also more than $95 \%$. In contrast, in the redshifted velocity range, there is almost no missing flux at $V_{\mathrm{LSR}}>8.4 \mathrm{~km} \mathrm{~s}^{-1}$. If the large-scale ambient gas is optically thick at the low velocities of $V_{\mathrm{LSR}} \sim 5-7 \mathrm{~km} \mathrm{~s}^{-1}$, our ALMA observations cannot detect the ${ }^{13} \mathrm{CO}$ emission (if any) originating from the arc structures at these low velocities and would resolve out the optically thick surface of the large-scale ambient gas. Missing the emission at these low velocities can bias the observed mean velocity in the blueshifted arc structure to be more blueshifted. In Fig. 4 we denote the model velocity of the three-dimensional infalling and rotational motions at these positions. If the arc structures indeed follow the free-falling and rotational motions and show the higher velocity because of the missing flux, the actual spectra without the missing flux should be centered on this expected velocity from the model. This would imply that the actual line width of the blueshifted arc structure is $2 \mathrm{~km} \mathrm{~s}^{-1}$. On the other hand, there is less missing flux in the redshifted velocity range. The line width of the redshifted arc structure is narrower than $1.4 \mathrm{~km} \mathrm{~s}^{-1}$ at the offset of F. $(0$ '.7, -3 '.5), where it is less strongly blended with the low-velocity outflow. Thus, the line width of the blueshifted arc structure is not expected to be as wide as $2 \mathrm{~km} \mathrm{~s}^{-1}$, assuming that the blue- and redshifted arc structures have the same origin and similar physical conditions. The high observed mean velocity in the northwestern part of the blueshifted arc structure is probably not purely caused by the missing flux. Nevertheless, adding short-spacing data to fully recover the structures at low velocities with a high angular resolution is essential to examine this possibility. 


\section{Discussion}

Our ALMA observations reveal the arc structures in the ${ }^{13} \mathrm{CO}$ emission that are connected to the protoplanetary disk around HL Tau (Figs. 3b and c). The overall velocity features of the arc structures can be explained with three-dimensional infalling and rotational motions (Fig. 9a). However, the observed relative velocity in the northwestern part of the blueshifted arc structure is higher than the expectation from the free-fall motion, and the deviation is as large as $0.8 \mathrm{~km} \mathrm{~s}^{-1}$, corresponding to $\sim 60-70 \%$ of the expected free-fall velocity (Fig. 9). Thus, its kinematics cannot be fully explained with the conventional model of infalling and rotational motions (e.g., Ulrich 1976). This suggests that the gravity of HL Tau is insufficient to accelerate the infalling gas to the observed relative velocity. If the observed arc structures are indeed infalling toward the protoplanetary disk around HL Tau, a force in addition to the gravity of HL Tau is required to drive the infalling motion.

The ${ }^{13} \mathrm{CO}(1-0)$ map with a size of $6^{\prime}$ toward HL Tau obtained by combining BIMA and NRAO $12 \mathrm{~m}$ data shows a shell with a size of $2^{\prime} \times 1 ' 5(\sim 11000$ au in radius $)$ centered on XZ Tau that is located $25^{\prime \prime}$ east of HL Tau (Welch et al. 2000). This large-scale shell is interpreted as an expanding bubble with an expanding velocity of $1.2 \mathrm{~km} \mathrm{~s}^{-1}$ based on its velocity structures observed in the ${ }^{13} \mathrm{CO}(1-0)$ emission, and the expansion is suggested to be driven by XZ Tau (Welch et al. 2000). XZ Tau is a binary system and classified as a classical T Tauri star. Observations with the Hubble Space Telescope reveal several concentric elongated bubbles on a $10^{\prime \prime}$ scale around XZ Tau, suggesting that XZ Tau has episodically ejected wide-angle outflows (Krist et al. $1999,2008)$. The molecular outflows in XZ Tau have been observed in the CO (1-0) emission on a $10^{\prime \prime}$ scale with ALMA, and the observed velocity features also hint at wide-angle expansion (Zapata et al. 2015). HL Tau is located at the wall of the largescale expanding shell. Thus, the protostellar envelope around HL Tau can be compressed by the expansion. The single-dish observations indeed show that the systemic velocity of the largescale ambient gas is different from that of HL Tau (Monin et al. 1996; Cabrit et al. 1996). This suggests that the current protostellar envelope around HL Tau could have relative motion with respect to HL Tau, possibly due to the compression by the expanding shell. The dynamical timescale of the expansion can be estimated as the radius of the expanding shell (11000 au) over the expanding velocity $\left(1.2 \mathrm{~km} \mathrm{~s}^{-1}\right)$ to be $4 \times 10^{4} \mathrm{yr}$. This is shorter than the typical lifetime of the Class 0 and I stage ( $10^{5} \mathrm{yr}$; Enoch et al. 2009). Thus, initially HL Tau could form out of a protostellar envelope having a $V_{\text {sys }}$ of $7 \mathrm{~km} \mathrm{~s}^{-1}$, as we measured from the disk rotation (Sect. 4.1). Later, the protostellar envelope could be compressed by the expanding shell driven by XZ Tau and have motions relative to HL Tau, resulting in a different $V_{\text {sys }}$, and the outer envelope material could collapse toward HL Tau with a non-zero initial velocity. In this case, the infalling velocity can be higher than the expected free-fall velocity derived from the protostellar mass of HL Tau. The difference in the observed relative velocity and the expected freefall velocity in the northwestern part of the arc structures is $\lesssim 0.8 \mathrm{~km} \mathrm{~s}^{-1}$. This is comparable to the difference in the systemic velocities between HL Tau and the large-scale ambient gas, 0.4$0.7 \mathrm{~km} \mathrm{~s}^{-1}$, and is lower than the expanding velocity of the shell, $1.2 \mathrm{~km} \mathrm{~s}^{-1}$. Therefore, the scenario of the infalling envelope externally compressed by the expanding shell is able to explain the observed high velocity of the blueshifted arc structure, and the arc structures are infalling toward the protoplanetary disk around HL Tau.
The morphologies of the arc structures observed in HL Tau are much more strongly bent than the expected trajectories of material that is free-falling with a specific angular momentum of $1.9 \times 10^{-3} \mathrm{~km} \mathrm{~s}^{-1} \mathrm{pc}$, corresponding to a centrifugal radius of $100 \mathrm{au}$, toward HL Tau (see Fig. 2 in Tobin et al. 2012). Thus, the arc morphologies are probably not caused by the trajectory of the infalling material, unless the infalling material initially posses a much higher specific angular momentum than the central protoplanetary disk around HL Tau and loses its angular momentum through mechanisms such as magnetic braking within a 1000 au scale, making its velocity vector closer to the radial direction afterward. This is different from the case in the other Class I protostar L1489, where the morphologies and velocity structures of the infalling flows can be described with the conventional model of free-fall motion with a constant angular momentum (Yen et al. 2014). On the other hand, asymmetric arc structures are often present in numerical simulations of infalling envelopes. In magnetohydrodynamic (MHD) simulations including the effect of magnetic flux being decoupled from material accreted onto stars, the magnetic flux can accumulate and form regions with high magnetic pressure non-isotropically. These high-pressure regions can resist infalling material. Then infalling material flows along the boundary of the high-pressure region and can appear like arc structures (e.g., Fig. 6 in Zhao et al. 2011, and Fig. 6 in Krasnopolsky et al. 2012). In addition, in the MHD simulations, where the magnetic field and the rotational axis are misaligned, the flattened structures that are formed by mass accumulation perpendicular to the magnetic field direction can be twisted by the rotation. The twisted flattened structures can be seen as arcs or spirals after projection with certain inclination angles (e.g., Fig. 1 in Joos et al. 2012). Nevertheless, the magnetic field tends to slow down infall and rotation (e.g., Li et al. 2011). The observed arc structures with the velocity higher than the expected free-fall velocity cannot be explained by simply including the magnetic field effects. Asymmetric arc structures also form in the simulations incorporating turbulence, where the density distribution of an infalling envelope is perturbed by turbulence and can exhibit arc structures (e.g., Fig. 2 in Li et al. 2014). It is also possible that the morphologies of the arc structures are simply related to the initial density distribution and velocity structures of the infalling material, which are shaped by the expanding bubble driven by XZ Tau.

If the observed arc structures are indeed infalling toward the protoplanetary disk around HL Tau, the infalling timescale of the arc structures that are fully accreted onto the protoplanetary disk can be estimated as $r / V_{r}$. With $V_{r}$ and $r$ derived from the best-match model velocity field (Fig. 9a) and ignoring the additional acceleration caused by the external compression for simplicity, the mass-weighted mean infalling timescale is estimated to be $\sim 2600 \mathrm{yr}$. The mass of the arc structures is estimated to be $5.7 \times 10^{-3} M_{\odot}$ (Sect. 4.2). Then, the mass infalling rate onto the protoplanetary disk around HL Tau is estimated to be $5.7 \times 10^{-3} / 2600=2.2 \times 10^{-6} M_{\odot} \mathrm{yr}^{-1}$. We note that the mass of the arc structures is only approximately $10 \%$ of the total gas mass observed on a scale of a few thousand au around HL Tau. The mass accretion rate onto HL Tau can be estimated as $L_{\mathrm{bol}} R_{*} / G M_{*}$, where $R_{*}$ is the stellar radius of HL Tau. With $L_{\mathrm{bol}}=3.5-15 L_{\odot}$ (Robitaille et al. 2007), $M_{*}=1.8 M_{\odot}$, and $R_{*}=5 R_{\odot}$ (Palla \& Stahler 1991), the mass accretion rate is estimated to be $(0.3-1.4) \times 10^{-6} M_{\odot} \mathrm{yr}^{-1}$, assuming the bolometric luminosity of HL Tau fully originates from the gravitational energy released by the accretion. These mass infalling and accretion rates are significantly higher than 
those in solar-mass $\mathrm{T}$ Tauri stars, which have mass accretion rates of $10^{-7}-10^{-9} M_{\odot} \mathrm{yr}^{-1}$ (Beltrán \& de Wit 2016) and are comparable to those of Class 0 and I protostars (e.g., Yen et al. 2017). The estimated mass infalling rate onto the disk is comparable to or possibly higher than the estimated mass accretion rate onto HL Tau. Hence, the disk mass could gradually increase, and the disk around HL Tau may become gravitationally unstable because of the continuous mass infall from the envelope (Hennebelle et al. 2017; Tomida et al. 2017).

Alternatively, the arc structures observed in HL Tau can be outflowing rather than infalling. The arc structures show a velocity gradient along the disk minor axis, where the northeastern part is blueshifted and the southwestern part is redshifted. The direction of this velocity gradient is the same as that of the outflow in the CO lines observed with SMA and ALMA (Lumbreras \& Zapata 2014; ALMA Partnership et al. 2015; Klaassen et al. 2016). If the blue- and redshifted arc structures are located in front of and behind the disk plane, respectively, the observed velocity gradient can be explained with outflowing motion. The elongations of the arc structures are perpendicular to the outflow direction. The opening angle of the $\mathrm{CO}$ outflow is measured to be $60^{\circ}-90^{\circ}$ (Klaassen et al. 2016), and the infrared image also shows a V-shaped morphology with an opening angle of $60^{\circ}$ on a scale of $3^{\prime \prime}$ (Takami et al. 2007). The opening angle of the outflow is not as wide as the extension of the arc structures. In addition, the inner part of the arc structures has a higher velocity than the outer part. This velocity structure is different from the structure typically observed in molecular outflows, which shows higher velocities in outer parts (Shu et al. 1991, 2000; Lee et al. 2000). Thus, if the arc structures are indeed moving away from HL Tau, their outflowing motions are probably not due to the jets or wind launched by the central stardisk system. Finally, hydrodynamical simulations show that if a circumstellar disk is sufficiently massive and becomes gravitationally unstable, the disk can form several arc structures and/or fragment into gas clumps (e.g., Figs. 2 and 3 in Vorobyov 2016, Fig. 2 in Tomida et al. 2017, and Fig. 3 in Hennebelle et al. 2017). Arc structures similar to those in the simulations have been observed on scales of 500-1000 au around young stellar objects that are undergoing accretion outbursts with coronagraphic polarimetric imaging in the near-infrared (Liu et al. 2016). In the simulations, some of these gas clumps can be ejected away via their multi-body interaction, and the ejected gas clumps and the central disk are linked with arc structures (Vorobyov 2016). The ejected gas clumps can have velocities of a few times their escape velocities, and they have masses of tens of Jupiter mass, resulting in a high mass loss rate in a short period $\left(0.1-0.2 M_{\odot}\right.$ in $0.1 \mathrm{Myr}$; Vorobyov 2016). If the observed arc structures are gas clumps ejected by the protoplanetary disk around HL Tau due to the gravitational instability in the disk, it could explain the observed relative velocity, which is higher than the expected free-fall velocity. In addition, the mass of the observed arc structures is comparable to those of the ejected gas clumps in the simulations.

The analysis of the millimeter and submillimeter continuum emission in the protoplanetary disk around HL Tau observed with ALMA at angular resolutions of $00^{\prime \prime} 03-00^{\prime \prime 07}$ (5-10 au) indeed suggests that the disk can be gravitationally unstable at a radius larger than $\sim 50 \mathrm{au}$, and the gravitational instability is proposed as a possible mechanism to form hypothetical bodies that open gaps in the disk (Akiyama et al. 2016). Nevertheless, the analysis of the gravitational instability in the protoplanetary disk around HL Tau depends on the assumed gas-to-dust mass ratio, which was adopted to be 100 . With a gas-to-dust mass ratio of 100 , the disk mass was estimated to be $\sim 0.13 M_{\odot}$ (Kwon et al. 2011), which is $7 \%$ of the protostellar mass. This implies that the $\mathrm{C}^{18} \mathrm{O}$ abundance is 40 times lower than the typical ISM value based on the ratio between our estimated disk mass from the $\mathrm{C}^{18} \mathrm{O}$ emission and the disk mass of $0.13 M_{\odot}$ estimated from the continuum emission. A CO abundance lower than the ISM value by two orders of magnitude has been observed in the protoplanetary disk around TW Hya (Schwarz et al. 2016), and possibly also in several other disks (e.g., Ansdell et al. 2016). Therefore it is possible that the observed arc structures are formed by the gravitational instability in the protoplanetary disk and are moving away from HL Tau. In this case, the formation of the arc structures and the gaps in the protoplanetary disk around HL Tau could be linked.

\section{Summary}

We performed imaging and analyses on our ALMA cycle- 3 data of the ${ }^{13} \mathrm{CO}(2-1)$ and $\mathrm{C}^{18} \mathrm{O}(2-1)$ emission in the planet formation candidate HL Tau. The aim was to probe the gas kinematics in the protostellar envelope surrounding the protoplanetary disk and to investigate the interaction between the envelope and the disk. Our main results are summarized below.

1. The $\mathrm{C}^{18} \mathrm{O}$ emission primarily traces a compact component with a size of $2^{\prime \prime} \times 1$ '.5 (280 au $\left.\times 210 \mathrm{au}\right)$ around HL Tau. The ${ }^{13} \mathrm{CO}$ emission also shows a similar central component. The central components are oriented along the major axis of the protoplanetary disk and exhibit dominant velocity gradients along the disk major axis.

2. Two arc structures on a scale of 1000-2000 au connected to the central component are observed in the ${ }^{13} \mathrm{CO}$ emission. The blueshifted arc structure stretches from the northeast to the northwest, and the redshifted arc from the southwest to the southeast. The arc structures show overall velocity gradients along both the disk major and minor axes. The direction of the velocity gradient along the minor axis is consistent with the outflow and the infalling motion, and the velocity along the major axis is opposite to the rotational direction of the protoplanetary disk. The mass of the arc structures is estimated to be $5.7 \times 10^{-3} M_{\odot}$.

3. The rotational profile of the central component is measured to be $(2.44 \pm 0.07) \times\left(R_{\text {rot }} / R_{0}\right)^{-0.52 \pm 0.04} \mathrm{~km} \mathrm{~s}^{-1}$ in the $\mathrm{C}^{18} \mathrm{O}$ emission and $(2.47 \pm 0.12) \times\left(R_{\text {rot }} / R_{0}\right)^{-0.52 \pm 0.05} \mathrm{~km} \mathrm{~s}^{-1}$ in the ${ }^{13} \mathrm{CO}$ emission, and the systemic velocity is measured to be $V_{\mathrm{LSR}}=7.04 \pm 0.04 \mathrm{~km} \mathrm{~s}^{-1}$. This rotational profile is consistent with Keplerian rotation $(f=-0.5)$ within the uncertainty, suggesting that this central component traces the protoplanetary disk around HL Tau. The protostellar mass of HL Tau is estimated to be $1.8 \pm 0.3 M_{\odot}$ on the assumption that the inclination angle of the protoplanetary disk is $47^{\circ}$. The gas mass of the disk is estimated to be $3.5 \times 10^{-3} M_{\odot}$ with the ISM $\mathrm{C}^{18} \mathrm{O}$ abundance. This is comparable to the dust mass of the disk, $(0.3-3) \times 10^{-3} M_{\odot}$, estimated in previous studies of the continuum emission, suggesting a low gas-todust mass ratio of $<10$ or a low $\mathrm{C}^{18} \mathrm{O}$ abundance of $<10^{-8}$ in the protoplanetary disk around HL Tau.

4. Comparison between the moment 1 maps of the arc structures observed in the ${ }^{13} \mathrm{CO}$ emission with the models of different gas motions suggests that the kinematics of the arc structures cannot be fully explained with the infalling and (counter-)rotational motions in the flattened envelope around HL Tau. The overall velocity structures can be better explained with the three-dimensional infalling and rotational 
motions, where the blue- and redshifted arc structures are located behind and in front of the disk plane, respectively. However, the observed mean velocity in the northwest part of the blueshifted arc structure is more blueshifted than the expectation from the model, suggesting that the observed velocity is higher than the expected free-fall velocity. The velocity excess is as high as $0.8 \mathrm{~km} \mathrm{~s}^{-1}$ along the line of sight, corresponding to $\sim 60-70 \%$ of the expected free-fall velocity.

5. If the arc structures are indeed infalling flows toward the protoplanetary disk around HL Tau, its infalling velocity higher than the expected free-fall velocity can be explained with the external compression. HL Tau is located at the edge of an expanding shell driven by a nearby $\mathrm{T}$ Tauri star, XZ Tau. The systemic velocity of the ambient gas on a scale of $0.1 \mathrm{pc}$ is $V_{\mathrm{LSR}}=6.3-6.6 \mathrm{~km} \mathrm{~s}^{-1}$, which is more strongly blueshifted than the velocity of the central disk. Thus, the protostellar envelope could have relative motion with respect to HL Tau because of the external compression by the expanding shell, resulting in the higher infalling velocity. In this scenario, the mass infalling rate onto the protoplanetary disk is estimated to be $2.5 \times 10^{-6} M_{\odot} \mathrm{yr}^{-1}$, comparable to or higher than the mass accretion rate onto HL Tau, $(0.3-1.4) \times 10^{-6} M_{\odot} \mathrm{yr}^{-1}$. Thus, the protoplanetary disk around HL Tau could gradually increase its mass.

6. Alternatively, the motions of the arc structures can be outflowing instead of infalling, if the blue- and redshifted arc structures are located in front of and behind the disk plane. The previous ALMA observations in the (sub-)millimeter continuum emission toward the protoplanetary disk around HL Tau suggest that the outer part $(r>50 \mathrm{au})$ of the disk can be gravitational unstable. The instability can cause a protoplanetary disk to form arc structures and gas clumps with masses of tens of Jupiter mass. Some of the gas clumps can be ejected via multi-body interaction and move away from the disk at velocities of a few times their escaping velocities, and the ejected gas clumps and the disk are connected with arc structures. Thus, the outflowing motion driven by the instability could explain the high velocity in the northwestern part of the blueshifted arc structure.

Acknowledgements. This paper makes use of the following ALMA data ADS/JAO.ALMA\#2015.1.00551.S. ALMA is a partnership of ESO (representing its member states), NSF (USA) and NINS (Japan), together with NRC (Canada), NSC and ASIAA (Taiwan), and KASI (Republic of Korea), in cooperation with the Republic of Chile. The Joint ALMA Observatory is operated by ESO, auI/NRAO and NAOJ. We thank all the ALMA staff supporting this work We acknowledge Bo Zhao for fruitful discussion on formation of arc structures in numerical simulations. S.T. acknowledges a grant from the Ministry of Science and Technology (MOST) of Taiwan (MOST 102-2119-M-001-012-MY3) and JSPS KAKENHI Grant Number JP16H07086, in support of this work. N.H is supported by the grant from MOST (MoST 105-2112-M-001-026). S.M. acknowledges a grant from MOST (MoST 103-2112-M-001-032-MY3). K.D.K was supported by the Polish National Science Centre MAESTRO grant DEC2012/06/A/ST9/00276.

\section{References}

Akiyama, E., Muto, T., Kusakabe, N., et al. 2015, ApJ, 802, L17 Akiyama, E., Hasegawa, Y., Hayashi, M., \& Iguchi, S. 2016, ApJ, 818, 158 ALMA Partnership, Brogan, C. L., Pérez, L. M., et al. 2015, ApJ, 808, L3 Andrews, S. M., Wilner, D. J., Zhu, Z., et al. 2016, ApJ, 820, L40

Anglada, G., López, R., Estalella, R., et al. 2007, AJ, 133, 2799 Ansdell, M., Williams, J. P., van der Marel, N., et al. 2016, ApJ, 828, 46 Aso, Y., Ohashi, N., Saigo, K., et al. 2015, ApJ, 812, 27
Beckwith, S. V. W., Sargent, A. I., Chini, R. S., \& Guesten, R. 1990, AJ, 99, 924

Beltrán, M. T., \& de Wit, W. J. 2016, A\&ARv, 24, 6

Brittain, S. D., Rettig, T. W., Simon, T., \& Kulesa, C. 2005, ApJ, 626, 283

Cabrit, S., Guilloteau, S., Andre, P., et al. 1996, A\&A, 305, 527

Carrasco-González, C., Henning, T., Chandler, C. J., et al. 2016, ApJ, 821, L16 D'Alessio, P., Calvet, N., \& Hartmann, L. 1997, ApJ, 474, 397

Dipierro, G., Price, D., Laibe, G., et al. 2015, MNRAS, 453, L73 Dong, R., \& Fung, J. 2017, ApJ, 835, 146

Dong, R., Fung, J., \& Chiang, E. 2016, ApJ, 826, 75

Dong, R., Zhu, Z., \& Whitney, B. 2015, ApJ, 809, 93

Dutrey, A., Guilloteau, S., \& Simon, M. 1994, A\&A, 286, 149

Enoch, M. L., Evans, N. J., II, Sargent, A. I., \& Glenn, J. 2009, ApJ, 692, 973

Ginski, C., Stolker, T., Pinilla, P., et al. 2016, A\&A, 595, A112

Hayashi, M., \& Pyo, T.-S. 2009, ApJ, 694, 582

Hayashi, M., Ohashi, N., \& Miyama, S. M. 1993, ApJ, 418, L71

Hennebelle, P., Lesur, G., \& Fromang, S. 2017, A\&A, 599, A86 Isella, A., Guidi, G., Testi, L., et al. 2016, Phys. Rev. Lett., 117, 251101

Jin, S., Li, S., Isella, A., Li, H., \& Ji, J. 2016, ApJ, 818, 76

Joos, M., Hennebelle, P., \& Ciardi, A. 2012, A\&A, 543, A128

Kanagawa, K. D., Muto, T., Tanaka, H., et al. 2015, ApJ, 806, L15

Kanagawa, K. D., Muto, T., Tanaka, H., et al. 2016, PASJ, 68, 43

Kenyon, S. J., Dobrzycka, D., \& Hartmann, L. 1994, AJ, 108, 1872

Klaassen, P. D., Mottram, J. C., Maud, L. T., \& Juhasz, A. 2016, MNRAS, 460, 627

Konishi, M., Grady, C. A., Schneider, G., et al. 2016, ApJ, 818, L23

Krasnopolsky, R., Li, Z.-Y., Shang, H., \& Zhao, B. 2012, ApJ, 757, 77

Krist, J. E., Stapelfeldt, K. R., Burrows, C. J., et al. 1999, ApJ, 515, L35

Krist, J. E., Stapelfeldt, K. R., Hester, J. J., et al. 2008, AJ, 136, 1980

Kwon, W., Looney, L. W., \& Mundy, L. G. 2011, ApJ, 741, 3

Lee, C.-F., Mundy, L. G., Reipurth, B., Ostriker, E. C., \& Stone, J. M. 2000, ApJ, 542,925

Li, Z.-Y., Krasnopolsky, R., \& Shang, H. 2011, ApJ, 738, 180

Li, Z.-Y., Krasnopolsky, R., Shang, H., \& Zhao, B. 2014, ApJ, 793, 130

Liu, H. B., Takami, M., Kudo, T., et al. 2016, Sci. Adv., 2, e1500875

Loinard, L. 2013, in Advancing the Physics of Cosmic Distances, IAU Symp., 289,36

Lumbreras, A. M., \& Zapata, L. A. 2014, AJ, 147, 72

Machida, M. N., Inutsuka, S.-I., \& Matsumoto, T. 2010, ApJ, 724, 1006

Men'shchikov, A. B., Henning, T., \& Fischer, O. 1999, ApJ, 519, 257

McMullin, J. P., Waters, B., Schiebel, D., et al. 2007, Astronomical Data Analysis Software and Systems XVI, eds. R. A. Shaw, F. Hill, \& D. J. Bell (San Francisco, CA: ASP), ASP Conf. Ser., 376, 127

Mendoza, S., Cantó, J., \& Raga, A. C. 2004, Rev. Mex. Astron. Astrofis., 40, 147

Momose, M., Morita, A., Fukagawa, M., et al. 2015, PASJ, 67, 83

Monin, J.-L., Pudritz, R. E., \& Lazareff, B. 1996, A\&A, 305, 572

Movsessian, T. A., Magakian, T. Y., Bally, J., et al. 2007, A\&A, 470, 605

Movsessian, T. A., Magakian, T. Y., \& Moiseev, A. V. 2012, A\&A, 541, A16

Ohashi, N., Saigo, K., Aso, Y., et al. 2014, ApJ, 796, 131

Okuzumi, S., Momose, M., Sirono, S.-I., Kobayashi, H., \& Tanaka, H. 2016, ApJ, 821, 82

Oya, Y., Sakai, N., López-Sepulcre, A., et al. 2016, ApJ, 824, 88

Palla, F., \& Stahler, S. W. 1991, ApJ, 375, 288

Perrot, C., Boccaletti, A., Pantin, E., et al. 2016, A\&A, 590, L7

Pinte, C., Dent, W. R. F., Ménard, F., et al. 2016, ApJ, 816, 25

Rapson, V. A., Kastner, J. H., Millar-Blanchaer, M. A., \& Dong, R. 2015, ApJ, 815, L26

Robitaille, T. P., Whitney, B. A., Indebetouw, R., \& Wood, K. 2007, ApJS, 169, 328

Sakai, N., Oya, Y., Sakai, T., et al. 2014a, ApJ, 791, L38

Sakai, N., Sakai, T., Hirota, T., et al. 2014b, Nature, 507, 78

Sakai, N., Oya, Y., López-Sepulcre, A., et al. 2016, ApJ, 820, L34

Sargent, A. I., \& Beckwith, S. 1987, ApJ, 323, 294

Sargent, A. I., \& Beckwith, S. V. W. 1991, ApJ, 382, L31

Schwarz, K. R., Bergin, E. A., Cleeves, L. I., et al. 2016, ApJ, 823, 91

Shirley, Y. L., Evans, N. J., II, Rawlings, J. M. C., \& Gregersen, E. M. 2000, ApJS, 131, 249

Shu, F. H., Ruden, S. P., Lada, C. J., \& Lizano, S. 1991, ApJ, 370, L31

Shu, F. H., Najita, J. R., Shang, H., \& Li, Z.-Y. 2000, Protostars and Planets IV (Tucson: University of Arizona Press), 789

Smith, R. L., Pontoppidan, K. M., Young, E. D., \& Morris, M. R. 2015, ApJ, 813,120

Takahashi, S. Z., \& Inutsuka, S.-I. 2014, ApJ, 794, 55

Takami, M., Beck, T. L., Pyo, T.-S., McGregor, P., \& Davis, C. 2007, ApJ, 670, L33

Tamayo, D., Triaud, A. H. M. J., Menou, K., \& Rein, H. 2015, ApJ, 805, 100

Testi, L., Skemer, A., Henning, T., et al. 2015, ApJ, 812, L38 
H.-W. Yen et al.: Arc structures connected to the disk of HL Tau

Tobin, J. J., Hartmann, L., Bergin, E., et al. 2012, ApJ, 748, 16

Tomida, K., Machida, M. N., Hosokawa, T., Sakurai, Y., \& Lin, C. H. 2017, ApJ, 835, L11

Ulrich, R. K. 1976, ApJ, 210, 377

van der Plas, G., Wright, C. M., Ménard, F., et al. 2017, A\&A, 597, A32

Visser, R., van Dishoeck, E. F., Doty, S. D., \& Dullemond, C. P. 2009, A\&A, 495, 881

Visser, R., Doty, S. D., \& van Dishoeck, E. F. 2011, A\&A, 534, A132

Vorobyov, E. I. 2010, ApJ, 723, 1294

Vorobyov, E. I. 2011, ApJ, 729, 146

Vorobyov, E. I. 2016, A\&A, 590, A115

Welch, W. J., Hartmann, L., Helfer, T., \& Briceño, C. 2000, ApJ, 540, 362

Wilson, T. L., \& Rood, R. 1994, ARA\&A, 32, 191
Yen, H.-W., Takakuwa, S., Ohashi, N., \& Ho, P. T. P. 2013, ApJ, 772, 22

Yen, H.-W., Takakuwa, S., Ohashi, N., et al. 2014, ApJ, 793, 1

Yen, H.-W., Liu, H. B., Gu, P.-G., et al. 2016, ApJ, 820, L25

Yen, H.-W., Koch, P. M., Takakuwa, S., et al. 2017, ApJ, 834, 178

Yorke, H. W., \& Bodenheimer, P. 1999, ApJ, 525, 330

Zapata, L. A., Galván-Madrid, R., Carrasco-González, C., et al. 2015, ApJ, 811, L4

Zhang, K., Blake, G. A., \& Bergin, E. A. 2015, ApJ, 806, L7

Zhang, K., Bergin, E. A., Blake, G. A., et al. 2016, ApJ, 818, L16

Zhao, B., Li, Z.-Y., Nakamura, F., Krasnopolsky, R., \& Shang, H. 2011, ApJ, 742,10

Zhu, Z., Hartmann, L., Nelson, R. P., \& Gammie, C. F. 2012, ApJ, 746, 110 


\section{Appendix A: Measuring ridges of contour lines}

The ridges of the contour lines were measured as follows. First we set a center, approximately the center of the curvature of the arc structures. Then we extracted a series of intensity profiles along the lines passing through that center in steps of PA of every $10^{\circ}-20^{\circ}$. For each intensity profile, we fit a Gaussian profile to measure the peak position. These peak positions denote the ridges of the contour lines shown in Fig. 3. The selected center for the arc structures at the blueshifted medium-high velocity is $\left(-1^{\prime \prime} .5,00^{\prime \prime} .5\right)$; at the redshifted medium-high velocity, the center is $\left(1^{\prime \prime} 0,-1{ }^{\prime \prime} 5\right)$; and at the redshifted medium-low velocity the center is $\left(0^{\prime \prime} .5,-1^{\prime \prime} .5\right)$. The arc structure at the blueshifted medium-low velocity is more extended, and two centers were selected at $\left(-4^{\prime \prime} 5,5^{\prime \prime} .0\right)$ and $\left(1^{\prime \prime} 0,2^{\prime \prime} .0\right)$ to extract the intensity profiles to cover the whole structure. We tested and confirm that the derived ridges are not sensitive to the choice of the centers.

\section{Appendix B: Velocity channel maps of the ${ }^{13} \mathrm{CO}$ emission}

Figures B.1 and B.2 present the entire velocity channel maps of the ${ }^{13} \mathrm{CO}$ emission at medium velocities, where the arc structures are observed, and at low velocities, where the extended structures are observed.

\section{Appendix C: Kinematical models for the arc structures}

\section{C.1. Infall and rotation model}

To compute this model, we adopt the geometrically thin approximation and assume that the arc structures are free-falling toward HL Tau and rotating with a constant specific angular momentum of $1.9 \times 10^{-3} \mathrm{~km} \mathrm{~s}^{-1} \mathrm{pc}$, the same as that at the outer disk radius. The model velocity profiles are adopted to be

$V_{\phi}=V_{\phi}(100 \mathrm{au}) \times\left(\frac{r}{100 \mathrm{au}}\right)^{-1}$,

$V_{r}=\sqrt{\frac{2 G M_{*}}{r}}$,

where $V_{\phi}$ and $V_{r}$ are the azimuthal and radial velocities, $G$ is the gravitational constant, $M_{*}$ is the measured protostellar mass of HL Tau $\left(1.8 M_{\odot}\right)$, and $V_{\phi}(100 \mathrm{au})$ is $3.9 \mathrm{~km} \mathrm{~s}^{-1}$ from the measured rotational profile (Sect. 4.1).

\section{C.2. Infall and counter-rotation model}

In this model, we also adopt the geometrically thin approximation, and we assume that the arc structures are free-falling toward HL Tau and rotating in the counter direction with respect to the disk rotation. The observed relative velocity in the northwestern part of the blueshifted arc structure is $\sim 1.5 \mathrm{~km} \mathrm{~s}^{-1}$ at a projected radius of $7^{\prime \prime}(\sim 1000 \mathrm{au}$; Fig. 7$)$. When we attribute this relative velocity to the counter-rotation, it corresponds to a rotational velocity of $2.1 \mathrm{~km} \mathrm{~s}^{-1}$ after correcting the inclination angle with the geometrically thin approximation, and this is higher than the expected free-fall velocity at a radius of $1000 \mathrm{au}$, which is $1.8 \mathrm{~km} \mathrm{~s}^{-1}$. Thus, the arc structures are expected to be rotationally supported if the angular momentum is conserved. If the arc structures are indeed infalling and rotating in the counter direction, their angular momentum has to be dissipated as they fall toward the center. To mimic the angular momentum dissiplation, we assume the model velocity profile of the counter rotation to be

$V_{\phi}=V_{\phi}(1000 \mathrm{au}) \times\left(\frac{r}{1000 \mathrm{au}}\right)$,

where $V_{\phi}(1000 \mathrm{au})=2.1 \mathrm{~km} \mathrm{~s}^{-1}$. The model velocity profile of the infalling motion is the same as Eq. (C.2).

\section{C.3. Three-dimensional infall and rotation model}

The model velocity profiles of three-dimensional infalling and rotational motions were adopted from Ulrich (1976) and Mendoza et al. (2004) as

$V_{r}=-\sqrt{\frac{G M_{*}}{r}} \cdot \sqrt{1+\frac{\cos \theta}{\cos \theta_{0}}}$,

$V_{\theta}=\sqrt{\frac{G M_{*}}{r}} \cdot \frac{\left(\cos \theta_{0}-\cos \theta\right)}{\sin \theta} \cdot \sqrt{1+\frac{\cos \theta}{\cos \theta_{0}}}$,

$V_{\phi}=\sqrt{\frac{G M_{*}}{r}} \cdot \frac{\sin \theta_{0}}{\sin \theta} \cdot \sqrt{1-\frac{\cos \theta}{\cos \theta_{0}}}$,

where $\theta$ is the angle between the radius of infalling gas and the polar axis, $\theta_{0}$ is the initial $\theta$ of infalling gas, and $V_{\theta}$ is the velocity along the $\theta$ direction. This model describes material free-falling toward a point mass with zero total energy and a conserved angular momentum. The $V_{\text {los }}$ of three-dimensional infalling and rotational motions after projection can be computed as

$V_{\text {los }}=\left(\left(V_{r} \sin \theta-V_{\theta} \cos \theta\right) \sin \phi+V_{\phi} \cos \phi\right) \sin i$

where $\phi$ is the azimuthal angle. The inclination angle and the rotational axis of the three-dimensional model are adopted to be the same as those of the protoplanetary disk around HL Tau.

Along a given line of sight, the infalling gas located closer to the disk plane has a higher infalling velocity because of the smaller distance to the star, while its velocity vector is almost on the disk major axis and is perpendicular to the line of sight. The two effects compensate for each other and result in a low $\left|V_{\text {los }}\right|$. On the other hand, the infalling gas located farther away from the disk plane has a lower infalling velocity and a velocity vector more parallel to the line of sight. That also results in a low $\left|V_{\text {los }}\right|$. Hence, there is a maximum $\left|V_{\text {los }}\right|$ induced by the infalling and rotational motions along a given light of sight, where the distance of the infalling gas to the disk plane and the angle between its velocity vector and the line of sight are moderate. To generate the model moment 1 map of the three-dimensional infalling and rotational motions, we assume that the arc structures are composed of an assemble of infalling and rotating particles, and each particle can be located anywhere along the line of sight. Then, for a given pixel in the model map, we compute all the possible $V_{\text {los }}$ of these infalling and rotating particles along the line of sight with Eq. (C.7). When the $V_{\text {los }}+V_{\text {sys }}\left(=V_{\text {LSR }}\right)$ of a particle matches the observed velocity at the same position, its $V_{\text {los }}+V_{\text {sys }}$ is assigned to that pixel in the model moment 1 map. When none of the computed $V_{\text {los }}+V_{\text {sys }}$ can match the observed velocity, the $V_{\text {los }}+V_{\text {sys }}$ of the particle with the maximum $\left|V_{\text {los }}\right|$ along the line of sight is assigned to that pixel. In our calculation, $V_{\text {sys }}$ is adopted to be $7.04 \mathrm{~km} \mathrm{~s}^{-1}$, the same as the velocity of the central protoplanetary disk. With this process, we generate a model moment 1 map of the three-dimensional infalling and rotational motions that best matches the observations (Fig. 9a). 
H.-W. Yen et al.: Arc structures connected to the disk of HL Tau
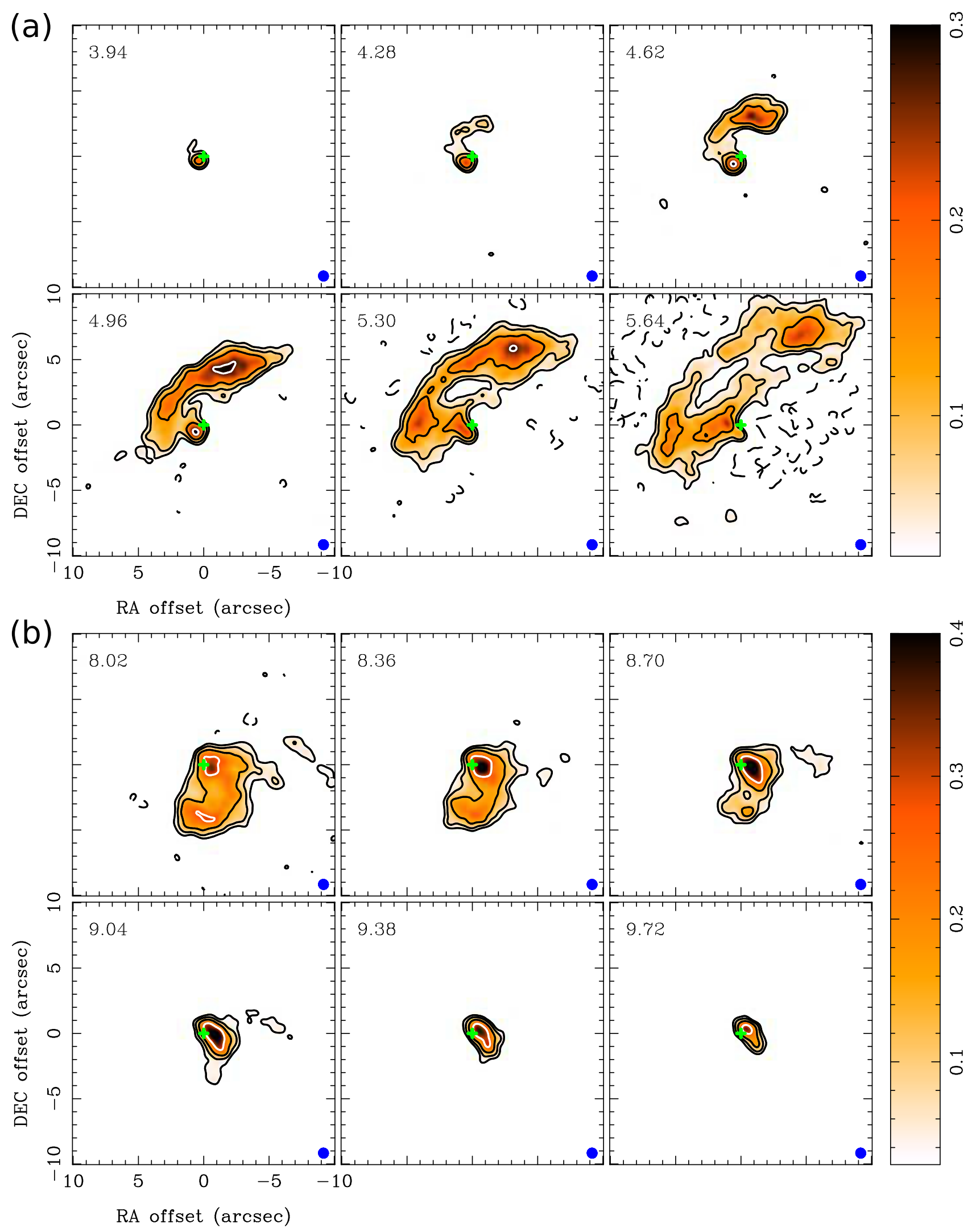

Fig. B.1. Velocity channel maps of the ${ }^{13} \mathrm{CO}$ emission in the blueshifted $(a)$ and redshifted $(b)$ medium-velocity ranges. Contour levels are $5 \sigma$, $10 \sigma, 20 \sigma$, and $40 \sigma$, where $1 \sigma$ is $7 \mathrm{mJy} \mathrm{Beam}^{-1}$. Color scales are in units of Jy Beam ${ }^{-1}$. Crosses denote the position of HL Tau. Filled ellipses present the size of the synthesized beam. The LSR velocity in units of $\mathrm{km} \mathrm{s}^{-1}$ of each channel is labeled in the upper left corner in each panel. 


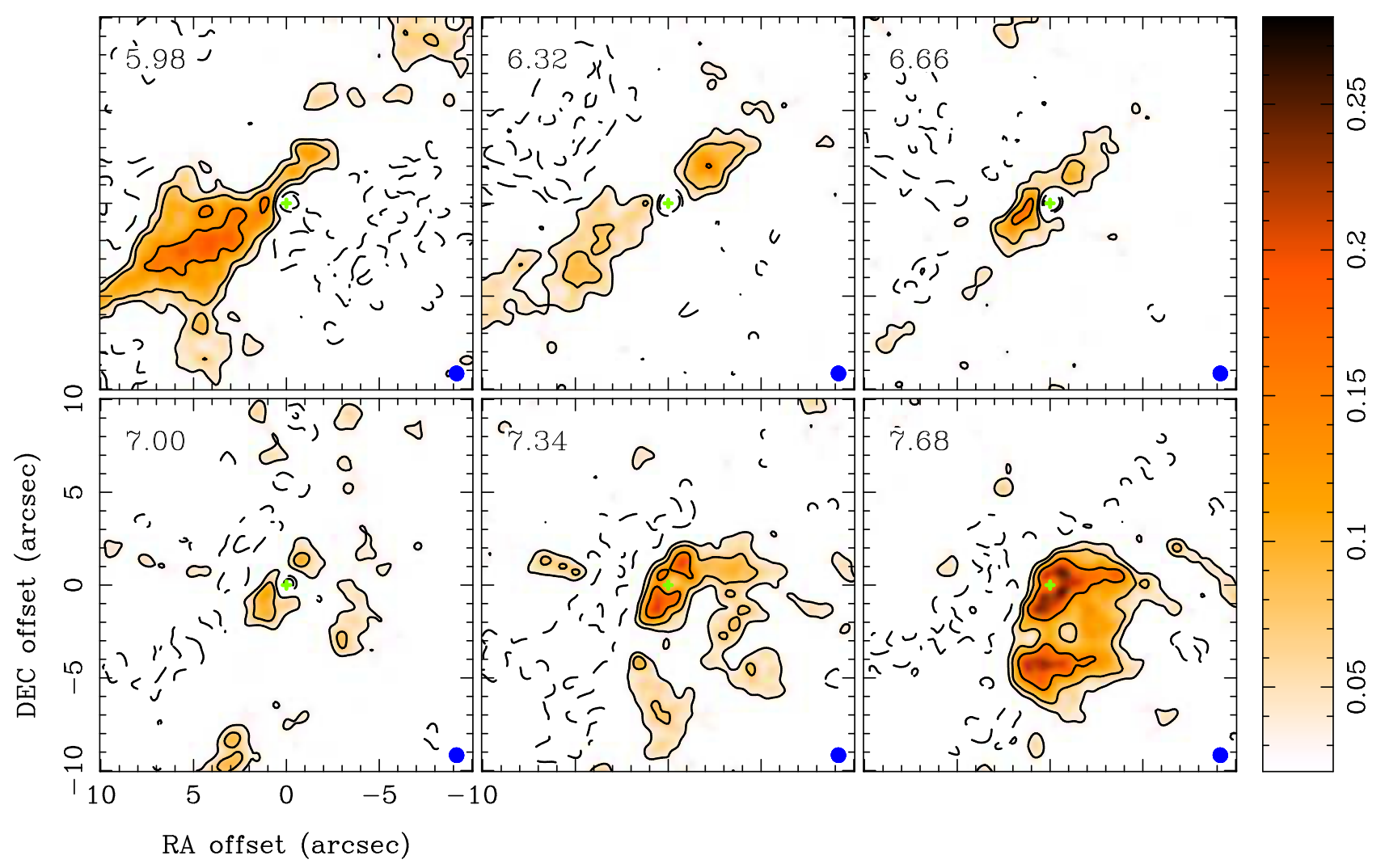

Fig. B.2. Same as Fig. B.1, but for the low-velocity range. 